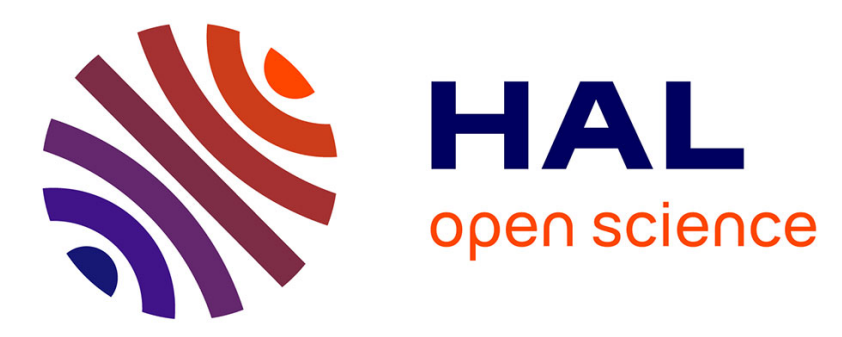

\title{
The representation of conditional relative importance between criteria
}

\author{
Christophe Labreuche, Michel Grabisch
}

\section{To cite this version:}

Christophe Labreuche, Michel Grabisch. The representation of conditional relative importance between criteria. Annals of Operations Research, 2007, 154 (1), pp.93-122. 10.1007/s10479-007-0184-2 . halshs-00187184

\section{HAL Id: halshs-00187184 \\ https://shs.hal.science/halshs-00187184}

Submitted on 13 Nov 2007

HAL is a multi-disciplinary open access archive for the deposit and dissemination of scientific research documents, whether they are published or not. The documents may come from teaching and research institutions in France or abroad, or from public or private research centers.
L'archive ouverte pluridisciplinaire HAL, est destinée au dépôt et à la diffusion de documents scientifiques de niveau recherche, publiés ou non, émanant des établissements d'enseignement et de recherche français ou étrangers, des laboratoires publics ou privés. 


\title{
The representation of conditional relative importance between criteria
}

\author{
Christophe Labreuche \\ Thales Research \& Technology \\ Domaine de Corbeville \\ 91404 Orsay Cedex, France
}

\author{
Michel Grabisch \\ University of Paris I \\ LIP6 - 4 place Jussieu \\ 75252 Paris, France
}

\begin{abstract}
We study in this paper the notion of conditional relative importance in a quantitative framework. Bi-capacities are shown to be suitable to represent such notion. We restrict ourself to the case when the relative importance of two criteria are conditional on a third being attractive or repulsive. We give two algorithms that enable to construct the neutral level from the preference relation.
\end{abstract}

Keywords: decision support systems, multiple criteria analysis, bi-capacity, Choquet integral, reference point.

\section{Introduction}

The stakes, consequences of managerial decisions over options under consideration are so important that many actors often take part in a Multi-Criteria Decision Aiding (MCDA) process. Actors can be experts that are consulted to give their opinion on a special aspect or attribute of the decision. Decision makers are also considered as actors of the decision, and their preferences are taken into account. This results in the presence of many viewpoints that are often conflicting.

It is very seldom that an option dominates the other ones over all or most of decision criteria. The selection of the best option is often performed by prioritizing the criteria. However, the semantics of the prioritization depends on the framework that is considered. We are interested here in options that are a good compromise between the criteria. More precisely, we look for compensation between criteria, that is that well-satisfied criteria can compensate ill-satisfied ones. It is well-known that quantitative Multi-Attribute Utility 
Theory (MAUT) [15] models such as the weighted sum are the best suited for representing compensation. The prioritization of the criteria is then performed, based on the notion of "relative importance" of one or several criteria compared to other ones.

An attribute can be more important than another one whatever the values of the remaining attributes may be. Consider the example of financial analysts in a bank that would like to decide on customers credit granting. Three factors have been retained: the debt ratio (denoted by $D$ ) given in $\%$, an indicator $P$ reflecting the behavior of the customer in the past (for instance the number of late payments) and the capital contribution ratio (denoted by $C$ ) given in \%. The main factor (i.e. the most important one) is the debt ratio. However, the preference of the analysts over $P$ and $C$ is not that simple. For a customer that has an attractive debt ratio, the contribution rate is not so important so that $P$ is more important than $C$. On the other hand, for a customer that has a bad debt ratio, there is relative substitutability between $D$ and $P$ so that the analysts hopes at least that the contribution ratio is good. Hence, $C$ becomes more important than $P$. We see in this example that the relative importance of one attribute compared to another one may depend on the value of a third attribute.

This type of statement is called "conditional relative importance" in TCPnets [7]. However, the semantics is purely qualitative in TCP-nets. More precisely, in this formalism, an attribute is more important than another one if we prefer a better value on the more important attribute whatever the value on the other attributes. We see that there is no compensation here.

In MAUT, the weighted sum cannot model conditional relative importance since the weight assigned to an attribute does not depend on the other attributes. Conditional relative importance is similar to the notion of interaction between criteria. In the literature, interaction is indeed often explained through conditional relative importance statements [12]. Here is a typical example :

(S1): If value w.r.t. criterion $i$ is very well-satisfied, then criterion $j^{+}$is more important than criterion $j^{-}$. If value w.r.t. criterion $i$ is very ill-satisfied, then criterion $j^{+}$is less important than criterion $j^{-}$.

Such statements are intuitive for actors. The Choquet integral has been shown to represent this type of statement [12]. The relative importance of criteria $j^{+}$and $j^{-}$is specified in statement (S1) only for the two extreme levels of performance (very good and very bad respectively) on criterion $i$. One wonders what happens for intermediate values on criterion $i$. One shall whenever possible specify the preferences between criteria $j^{+}$and $j^{-}$for all 
values of criterion $i$, as expressed in the conditional relative importance table for TCP-nets [7]. A generalization of statement (S1) is the following one :

(S2): If value w.r.t. criterion $i$ is "well-satisfied", then criterion $j^{+}$is more important than criterion $j^{-}$. If value w.r.t. criterion $i$ is "ill-satisfied", then criterion $j^{+}$is less important than criterion $j^{-}$.

In previous statement, the values judged as well-satisfied and ill-satisfied are supposed to be a partition of the scale. There exists thus on attribute $i$ a particular element called "neutral element" such that better elements are considered as well-satisfied and worse elements are considered as ill-satisfied for the actor. Hence, the scale underlying criterion $i$ is of bipolar nature. The Choquet fails to represent (S2) $[13,18]$.

We are interested in this paper in rule (S2) for two reasons. The first one is that it can be easily elicited by the actor. The second one is that we want to make an in-depth analysis of (S2) do to its close link with interaction between criteria. We investigate in Section 3 how (S2) can be represented by aggregation functions. We will show in Section 3 that (S2) cannot be strictly satisfied by a quantitative model under some natural restrictions (see Theorem Th6.1 below). This leads to considering a restriction of (S2) (see statement (S3) below).

Statement (S2) hinges on the values of the neutral level on attribute $i$. One often makes the assumption that the actor can provide such a reference point. We argue in this paper that this is not a trivial task for the actor. Unlike Decision Under Risk (DUR) in which the neutral level can often be identified as the zero-gain outcome, the neutral level has often no intrinsic existence in MCDA. We propose in Section 4 a method to determine the neutral elements. To our knowledge, the problem of determining the neutral level has not been extensively studied. There are some works in DUR (see for instance [19]). Utility functions often have a S-shape when there exists a reference point demarcating gains and losses [14]. This reference point can be assessed by determining the inflection point in the S-shape utility function [19]. In MCDA, the utility functions do not take in general such pre-defined form from which the value of the neutral value can be derived. However, our approach is that, if the actor is able to elicit (S2), one should be able to obtain an estimate of the neutral value by asking questions that challenge (S2). If (S2) is strictly satisfied, then for a given option, the value of the option w.r.t. attribute $i$ is better than the neutral value when criterion $j^{+}$ is more important than $j^{-}$. We make the opposite deduction when $j^{+}$is less important than $j^{-}$. This enables to iteratively guess the value of the 
neutral level by considering appropriate values on attribute $i$. Such simple procedure cannot be strictly applied for two reasons. The first one is that the questionnaire is not based on (S2) but on a restriction (namely (S3)). The second one is that the presence of the neutral element on attribute $i$ alters the perception the analyst can have on the actor's partial preferences over this attribute. We solve these two problems in Section 4.

\section{Preliminaries}

The set of all criteria is $N=\{1, \ldots, n\}$, and $X_{k}$ is the set of values that attribute $k \in N$ can take. Options are considered as elements of $X=$ $X_{1} \times \cdots \times X_{n}$. The preference relation over $X$ is denoted by $\succeq$. Considering two acts $x, y \in X$ and $A \subset N$, we use the notation $\left(x_{A}, y_{-A}\right)$ to denote the compound act $z \in X$ such that $z_{i}=x_{i}$ if $i \in A$ and $y_{i}$ otherwise.

Under some assumptions on $\succeq$ (in particular that $\succeq$ is a total pre-order and that one can construct from $\succeq$ partial preference relations $\succeq_{k}$ over each attribute $k$ ), the overall preference relation can be represented by an overall utility (i.e. $x \succeq y$ iff $U(x) \geq U(y))$ where $[16,6]$

$$
U(x)=F\left(u_{1}\left(x_{1}\right), \ldots, u_{n}\left(x_{n}\right)\right) .
$$

Partial utility function $u_{k}: X_{k} \rightarrow \mathbb{R}$ is a representation of $\succeq_{k}$, and $F$ is an aggregation function that is non-decreasing on each argument.

Since the aggregation functions we use are based on sums, the utility functions must be constructed as interval scales [16].

\subsection{Aggregation functions}

Statement (S2) uses the notion of relative importance. The relative importance of a criterion in an aggregation function $F$ is clearly defined when $F$ is a weighted sum $F\left(s_{1}, \ldots, s_{n}\right)=\sum_{k=1}^{n} w_{k} s_{k}$, where all weights $w_{k}$ are nonnegative and they sum up to one. The importance of criterion $k$ is then its associated weight $w_{k}$. More generally, if $F$ is piecewise linear, then there exists a partition of $\mathbb{R}^{n}$ such that $F$ is a weighted sum in each domain of the partition. The importance of a criterion is thus defined in each domain as its relative weight. Since any smooth function can be approximated by piecewise linear functions, this leads to defining the importance of criterion $k$ at point $s \in \mathbb{R}^{n}$ as the partial derivative $\frac{\partial F}{\partial s_{k}}(s)$ (since the weight of criterion $k$ in the

piecewise linear approximation converges to $\frac{\partial F}{\partial s_{k}}(s)$ when the size of the mesh describing the approximation tends to zero). Yet, this interpretation is not so simple for an actor. 
So, we will stick to piecewise linear aggregation functions so that the notion of relative importance clearly makes sense to the actor. He can elicit a statement such as (S2) only the notion of relative importance is clear to him. From measurement standpoint [16], piecewise linearity can be derived from the invariance to linear changes of the scales property [18]. The weight of criterion $l$ at point $s \in \Omega$ is denoted by $w_{l}(s)$ :

$$
F(s)=\sum_{l \in N} w_{l}(s) s_{l}
$$

where $w_{l}(s)$ is piecewise constant.

A piecewise linear function $F$ is characterized by a partition $\Phi(F)$ of $\mathbb{R}^{n}$ on which $F$ is a weighted sum on each element of the partition. Hence

$$
\Phi(F)=\left\{\Phi_{F}^{1}, \ldots, \Phi_{F}^{\phi(F)}\right\}
$$

where for all $l \in\{1, \ldots, \phi(F)\}$, there exists normalized weights $w_{1}^{\Phi_{F}^{l}}, \ldots, w_{n}^{\Phi_{F}^{l}}$ such that one has, for all $s \in \Phi_{F}^{l}, F(s)=\sum_{k \in N} w_{k}^{\Phi_{F}^{l}} s_{k}$. The partition is unique if each subset $\Phi_{F}^{l}$ is connected and

$$
\overline{\Phi_{F}^{l}} \cap \overline{\Phi_{F}^{l^{\prime}}} \neq \emptyset \Longrightarrow \exists k \in N, w_{k}^{\Phi_{F}^{l}} \neq w_{k}^{\Phi_{F}^{l^{\prime}}}
$$

\subsection{Bipolar and unipolar scales}

It may exists in $X_{k}$ a particular element or level $\mathbf{0}_{k}$, called neutral level, such that if $x_{k} \succ_{k} \mathbf{0}_{k}$, then $x_{k}$ is considered as "good", while if $x_{k} \succ_{k} \mathbf{0}_{k}$, then $x_{k}$ is considered as "bad" for the actor.

Such a neutral level exists whenever relation $\succeq_{k}$ corresponds to two opposite notions of common language. For example, this is the case when $\succeq_{k}$ means "more attractive than", "better than", etc., whose pairs of opposite notions are respectively "attractiveness/repulsiveness", and "good/bad". By contrast, relations "more satisfactory than", "more allowed than", "belongs more to category $C$ than" do not clearly exhibit a neutral level.

A scale is said to be bipolar if $X_{k}$ contains such a neutral level. A unipolar scale has no neutral level. As an example, preference statement (S2) implies that criterion $i$ is of bipolar nature.

\subsection{Aggregation of unipolar scales}

A capacity [8], also called fuzzy measure, is a set function $\nu: 2^{N} \rightarrow \mathbb{R}$ satisfying $\nu(\emptyset)=0, \nu(N)=1$, and $A \subset B$ implies $\nu(A) \leq \nu(B)$. In MCDA, $\nu(A)$ is interpreted as the overall assessment of the binary act $\left(1_{A}, 0_{-A}\right)$. 
The Choquet integral [8] defined w.r.t. a capacity $\nu$ has the following expression :

$$
\mathcal{C}_{\nu}\left(s_{1}, \ldots, s_{n}\right)=s_{\pi(1)} \nu(N)+\sum_{i=2}^{n}\left(s_{\pi(i)}-s_{\pi(i-1)}\right) \nu\left(A_{\pi(i)}\right)
$$

where $s_{\pi(1)} \leq s_{\pi(2)} \leq \cdots \leq s_{\pi(n)}, A_{\pi(i)}=\{\pi(i), \cdots, \pi(n)\}$ and $s_{1}, \ldots, s_{n} \in$ $\mathbb{R}_{+}$. We also have

$$
\mathcal{C}_{\nu}(s)=\sum_{i=1}^{n} s_{\pi(i)}\left[\nu\left(A_{\pi(i)}\right)-\nu\left(A_{\pi(i+1)}\right)\right] .
$$

Clearly, the Choquet integral w.r.t. a capacity is piecewise linear. Moreover, one has

$$
\Phi\left(\mathcal{C}_{\nu}\right)=\left\{\Omega_{\pi}, \pi \text { permutation on } N\right\}
$$

where $\Omega_{\pi}=\left\{s \in \mathbb{R}^{n}, s_{\pi(1)} \leq s_{\pi(2)} \leq \cdots \leq s_{\pi(n)}\right\}$.

\subsection{Aggregation on bipolar scales}

The Choquet integral has a natural extension to bipolar scales. The limitation of the Choquet integral w.r.t. capacities is that the overall evaluation at any point is computed from information coming only from the attractive part (i.e. the parameters of the capacity correspond to the overall assessment of the positive binary acts $\left.\left(1_{A}, 0_{-A}\right)\right)$. Hence, the notion of capacity is not suited to real bipolar scales. The idea is thus to generalize the notion of capacity. Let

$$
\mathcal{Q}(N)=\{(A, B) \in \mathcal{P}(N) \times \mathcal{P}(N) \mid A \cap B=\emptyset\}
$$

A bi-capacity $[13,18]$ is a function $\mu: \mathcal{Q}(N) \rightarrow \mathbb{R}$ satisfying $\mu(\emptyset, \emptyset)=0$, $\mu(N, \emptyset)=1, \mu(\emptyset, N)=-1, A \subset A^{\prime}$ implies $\mu(A, B) \leq \mu\left(A^{\prime}, B\right)$, and $B \subset B^{\prime}$ implies $\mu(A, B) \geq \mu\left(A, B^{\prime}\right)$. The last two properties depict increasingness. In MCDA, $\mu(A, B)$ is interpreted as the overall assessment of the ternary act $\left(1_{A},-1_{B}, 0_{-A \cup B}\right)$.

The Choquet integral w.r.t. a bi-capacity $\mu$ proposed in [13] is now given. For any $A \subset N, s \in \Sigma_{A}$,

$$
\mathcal{B C}_{\mu}(s):=\mathcal{C}_{\nu}\left(s_{A},-s_{-A}\right)=\mathcal{C}_{\nu}(|s|)
$$

where $\nu(C):=\mu(C \cap A, C \backslash A)$ and $\Sigma_{A}:=\left\{s \in \mathbb{R}^{n}, s_{A} \geq 0, s_{-A}<0\right\}$. Let $\tau$ be a permutation such that $\left|s_{\tau(1)}\right| \leq \ldots \leq\left|s_{\tau(n)}\right|$, and

$$
A_{\tau(i)}^{+}=\{\tau(i), \cdots, \tau(n)\} \cap A=\left\{\tau(j), \tau(j) \geq \tau(i) \text { and } s_{\tau(j)} \geq 0\right\}
$$




$$
A_{\tau(i)}^{-}=\{\tau(i), \cdots, \tau(n)\} \cap(N \backslash A)=\left\{\tau(j), \tau(j) \geq \tau(i) \text { and } s_{\tau(j)}<0\right\}
$$

Then one can write

$$
\mathcal{B C}_{\mu}(s)=\sum_{i=1}^{n}\left|s_{\tau(i)}\right|\left[\mu\left(A_{\tau(i)}^{+}, A_{\tau(i)}^{-}\right)-\mu\left(A_{\tau(i+1)}^{+}, A_{\tau(i+1)}^{-}\right)\right] .
$$

Clearly, the Choquet integral w.r.t. a bi-capacity is piecewise linear. Moreover, one has

$$
\Phi\left(\mathcal{B C}_{\mu}\right)=\left\{\Omega_{A, \tau}, A \subseteq N \text { and } \tau \text { permutation on } N\right\}
$$

where $\Omega_{A, \tau}=\left\{s \in \Sigma_{A},\left|s_{\tau(1)}\right| \leq \ldots \leq\left|s_{\tau(n)}\right|\right\}$.

\section{Representation of Statement (S2) by an ag- gregation function}

\subsection{Construction of the utility functions}

As we have seen in Section 2.2, statement (S2) clearly exhibits a bipolar behavior on criterion $i$. Due to commensurateness between the criteria, all criteria are considered as bipolar. Statement (S2) shows that there are interaction between criteria in preference relation over options. The construction of the utility functions $u_{k}$ is thus more complex than for the case where all criteria are independent (see for instance the utility independence assumption [15]). Utility functions $u_{k}$ have a priori no intrinsic meaning, and only make sense through the overall utility $U$ thanks to (1). When all criteria are independent, $U\left(x_{k}, z_{-k}\right)=F\left(u_{k}\left(x_{k}\right), u_{-k}\left(z_{-k}\right)\right)$ and $u_{k}\left(x_{k}\right)$ are two equivalent interval scales for any $z_{-k} \in X_{-k}$ fixed. This relation gives a sense to $u_{k}$. In this relation, the presence of $z_{-k}$ is not essential so that $u_{k}$ can be considered as a utility representation of a preference relation over attribute $X_{k}$ all else being equal (i.e. the value w.r.t. the other attributes being fixed to any value). The utility functions can thus be considered and constructed separately.

When there are some interactions between criteria, the "all else being equal" assumption does not hold anymore. The choice of the reference $z_{-k}$ from which the utility function is constructed becomes essential. For a given $z_{-k}$, if the following assumption

$$
\exists l \in\{1, \ldots, \phi(F)\}, \quad \forall x_{k} \in X_{k} \quad\left(u_{k}\left(x_{k}\right), u_{-k}\left(z_{-k}\right)\right) \in \Phi_{F}^{l}
$$

holds then utility function $u_{k}$ can be constructed as for the weighted sum since the options used in the construction of $u_{k}$ remain in the same domain 
$\Phi_{F}^{l}$ of linearity of $F$. Hence $F$ does not alter the perception of $u_{k}$ through $U\left(x_{k}, z_{-k}\right)$. For instance, if $F$ is a Choquet integral w.r.t. a capacity, $z_{-k}$ is considered at either the lowest or the highest satisfaction level on each attribute $l \neq k$ [17]. These two extreme values correspond to two reference levels. If $F$ is a Choquet integral w.r.t. a bi-capacity, the attractive and repulsive parts of $u_{k}$ must necessarily be constructed separately in order to have (5) (see the end of Section 2.4). The neutral level becomes an essential point in the construction of utility functions. Apart from the neutral level, one reference level is required on the attractive part and the repulsive one in order to normalize the scale. Hence three reference levels are necessary [18].

The actor is first asked to identify on each attribute $X_{k}$ a neutral element $\mathbf{0}_{k}$ that is considered as neither good nor bad [20]. Since statement (S2) clearly relies on the existence of such level on attribute $i$, one can assume that the actor who provides (S2) can identify the value of $\mathbf{0}_{i}$. We assume here that this neutral element can also be identified on the other attributes. We will give an alternative approach in Section 4. It is assumed furthermore that there exists an element denoted by $\mathbf{1}_{k}$ that is considered as good and completely satisfactory, even if more attractive elements could exist on this point of view [17]. The existence of such reference level comes from the theory of satisficing bounded rationality [21]. We assume finally that there exists an element denoted by $-\mathbf{1}_{k}$ that is considered as bad and unsatisfactory. Element $-\mathbf{1}_{k}$ is somehow symmetric to $\mathbf{1}_{k}$. More precisely, $\mathbf{- 1}_{k}$ corresponds to the same level of appreciation in the repulsive scale than $\mathbf{1}_{k}$ in the attractive scale. All levels have the same absolute meaning throughout the criteria, so we impose:

$$
\begin{aligned}
& u_{1}\left(-\mathbf{1}_{1}\right)=\cdots=u_{n}\left(-\mathbf{1}_{n}\right)=-1, \\
& u_{1}\left(\mathbf{0}_{1}\right)=\cdots=u_{n}\left(\mathbf{0}_{n}\right)=0 \\
& u_{1}\left(\mathbf{1}_{1}\right)=\cdots=u_{n}\left(\mathbf{1}_{n}\right)=1 .
\end{aligned}
$$

Since the attractive and repulsive values refer to different affect stimuli [22], it may be more appropriate to construct separately the positive and the negative parts of the partial utility functions in order to make the actor compare attractive values with repulsive ones. If $F$ satisfies

$$
\begin{array}{lll}
\exists l \in\{1, \ldots, \phi(F)\}, & \forall s_{k} \geq 0 & \left(s_{k}, 0_{-k}\right) \in \Phi_{F}^{l} \\
\exists l^{\prime} \in\{1, \ldots, \phi(F)\}, & \forall s_{k} \leq 0 & \left(s_{k}, 0_{-k}\right) \in \Phi_{F}^{l^{\prime}}
\end{array}
$$

then one can construct the utility function $u_{k}$ from $U\left(x_{k}, \mathbf{0}_{-k}\right)$ :

$$
\begin{array}{ll}
\forall x_{k} \succeq_{k} \mathbf{0}_{k}, \quad u_{k}\left(x_{k}\right)=\frac{U\left(x_{k}, \mathbf{0}_{-k}\right)-U\left(\mathbf{0}_{N}\right)}{U\left(\mathbf{1}_{k}, \mathbf{0}_{-k}\right)-U\left(\mathbf{0}_{N}\right)} \\
\forall x_{k} \preceq_{k} \mathbf{0}_{k}, \quad u_{k}\left(x_{k}\right)=\frac{U\left(x_{k}, \mathbf{0}_{-k}\right)-U\left(\mathbf{0}_{N}\right)}{U\left(\mathbf{0}_{N}\right)-U\left(-\mathbf{1}_{k}, \mathbf{0}_{-k}\right)}
\end{array}
$$


Relation (6) is satisfied by the Choquet integral w.r.t. a bi-capacity.

\subsection{General analysis}

Rule (S2) can be stated in the more precise form

(S2'): If the value w.r.t. criterion $i$ is attractive (i.e. $x_{i} \succ_{i} \mathbf{0}_{i}$ ) then criterion $j^{+}$is more important than criterion $j^{-}$. If the value w.r.t. criterion $i$ is repulsive (i.e. $x_{i} \prec_{i} \mathbf{0}_{i}$ ) then criterion $j^{+}$is less important than criterion $j^{-}$.

Aggregation functions are henceforth assumed to be continuous since a slight modification in the argument shall also result in a slight change in the overall utility [10]. The following lemma shows that the boundary of the domains on which a piecewise linear function is a weighted sum is not arbitrary.

Lemma 1 Let $F$ be a continuous piecewise linear function. Then necessarily, the boundaries of the domains of $\Phi(F)$ are hyperplanes.

Proof : Let $F$ be a piecewise linear function. Let $\Phi^{+}, \Phi^{-} \in \Phi(F)$ be two neighbor domains such that the boundary $\Gamma:=\overline{\Phi^{+}} \cap \overline{\Phi^{-}}$is a non empty surface described by

$$
G(s)=c .
$$

Let $I$ be the set of criteria on which $G$ depends. We denote by $w_{k}^{+}$the weight of $F$ in $\Phi^{+}$, and by $w_{k}^{-}$the weights of $F$ in $\Phi^{-}$. By (2), there exists $k \in N$ such that $w_{k}^{+} \neq w_{k}^{-}$.

By continuity of $F$ on $\Gamma$, we get for any $s \in \Gamma$,

$$
\sum_{l \in N}\left(w_{l}^{+}-w_{l}^{-}\right) s_{l}=0 .
$$

Let $s \in \Gamma$ and $l_{1} \in N \backslash I$. Then, since $G$ does not depend on $l_{1}$, there exists $s_{l_{1}}^{\prime} \neq s_{l_{1}}$ such that $\left(s_{l_{1}}^{\prime}, s_{-l_{1}}\right) \in \Gamma$. By (7), we obtain

$$
\left(w_{l_{1}}^{+}-w_{l_{1}}^{-}\right)\left(s_{l_{1}}^{\prime}-s_{l_{1}}\right)=0
$$

which gives

$$
\forall l_{1} \in N \backslash I, \quad w_{l_{1}}^{+}=w_{l_{1}}^{-} .
$$

Assume now by contradiction that $\Gamma$ is not an hyperplane. Then since $\Gamma$ is a surface, there exists $l_{1}, l_{2} \in I$ and $s_{-l_{1}, l_{2}}$ such that the restriction of 
$\Gamma$ to values $s_{-l_{1}, l_{2}}$ on criteria $N \backslash\left\{l_{1}, l_{2}\right\}$ is not a line. Thus there exists $s_{l_{1}}, s_{l_{1}}^{\prime}, s_{l_{1}}^{\prime \prime}, s_{l_{2}}, s_{l_{2}}^{\prime}, s_{l_{2}}^{\prime \prime}$ such that

$$
\left(s_{l_{1}}, s_{l_{2}}, s_{-l_{1}, l_{2}}\right) \in \Gamma,\left(s_{l_{1}}^{\prime}, s_{l_{2}}^{\prime}, s_{-l_{1}, l_{2}}\right) \in \Gamma,\left(s_{l_{1}}^{\prime \prime}, s_{l_{2}}^{\prime \prime}, s_{-l_{1}, l_{2}}\right) \in \Gamma
$$

and

$$
\frac{s_{l_{1}}^{\prime}-s_{l_{1}}}{s_{l_{1}}^{\prime \prime}-s_{l_{1}}} \neq \frac{s_{l_{2}}^{\prime}-s_{l_{2}}}{s_{l_{2}}^{\prime \prime}-s_{l_{2}}}
$$

Applying (7) to previous three points of $\Gamma$, we get

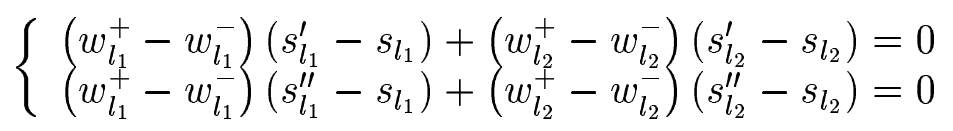

The only solution to previous system is the trivial one due to (9) :

$$
w_{l_{1}}^{+}=w_{l_{1}}^{-}, \quad w_{l_{2}}^{+}=w_{l_{2}}^{-} .
$$

Consider finally $l_{3} \in I \backslash\left\{l_{1}, l_{2}\right\}$. Let $s \in \Gamma$. There exists $s_{l_{1}}^{\prime} \neq s_{l_{1}}$ and $s_{l_{3}}^{\prime} \neq s_{l_{3}}$ such that $\left(s_{l_{1}}^{\prime}, s_{l_{3}}^{\prime}, s_{-l_{1}, l_{3}}\right) \in \Gamma$. By $(7)$,

$$
\left(w_{l_{1}}^{+}-w_{l_{1}}^{-}\right)\left(s_{l_{1}}^{\prime}-s_{l_{1}}\right)+\left(w_{l_{3}}^{+}-w_{l_{3}}^{-}\right)\left(s_{l_{3}}^{\prime}-s_{l_{3}}\right)=0 .
$$

From (10), we obtain that $w_{l_{3}}^{+}=w_{l_{3}}^{-}$.

We have shown that all weights $w^{+}$in $\Phi^{+}$are similar to that $w^{-}$in $\Phi^{-}$, which contradicts our previous assumption. Hence $\Gamma$ is necessarily a part of an hyperplane.

Next lemma shows that statement (S2') cannot be thoroughly modeled in all situations. Some restrictions will thus be made.

Theorem 1 There does not exist any aggregation function $F$ that is continuous and piecewise linear, for which (S2') is satisfied when criterion $i$ is the closest to the neutral level among criteria $i, j^{+}, j^{-}$.

\section{Proof :}

- Consider a piecewise linear aggregation function $F$ that is continuous. Let $\Phi^{+}, \Phi^{-} \in \Phi(F)$ be two neighbor domains such that the boundary $\Gamma:=\overline{\Phi^{+}} \cap \overline{\Phi^{-}}$is a non empty surface described by the hyper-plane (see Lemma 1)

$$
\sum_{l \in I_{\Phi^{+}, \Phi^{-}}} \alpha_{l} s_{l}=c
$$


for some $I_{\Phi^{+}, \Phi^{-}} \subset N$. We denote by $w_{k}^{+}$the weight of $F$ in $\Phi^{+}$, and by $w_{k}^{-}$the weights of $F$ in $\Phi^{-}$. Since $F$ is continuous, we get for all $s \in \Gamma$

$$
\sum_{l \in N} w_{l}^{+} s_{l}=\sum_{l \in N} w_{l}^{-} s_{l} .
$$

Let $\alpha \in \mathbb{R}^{n}$ having value $\alpha_{l}$ at component $l$ in $I_{\Phi^{+}, \Phi^{-}}$and value 0 at the other components. We have

$$
\forall s, s^{\prime} \in \Gamma \quad\left\{\begin{array}{l}
\sum_{l \in N}\left(w_{l}^{+}-w_{l}^{-}\right) \Delta s_{l}=0 \\
\sum_{l \in I_{\Phi^{+}, \Phi^{-}}} \alpha_{l} \Delta s_{l}=0
\end{array}\right.
$$

where $\Delta s_{l}:=s_{l}^{\prime}-s_{l}$. Let $l_{1} \in N \backslash I_{\Phi^{+}, \Phi^{-}}$. There exists $s_{l_{1}}^{\prime} \neq s_{l_{1}}$ such that $\left(s_{l_{1}}^{\prime}, s_{-l_{1}}\right) \in \Gamma$. Relation (11) gives $\left(w_{l_{1}}^{+}-w_{l_{1}}^{-}\right) \Delta s_{l_{1}}=0$. Since $\Delta s_{l_{1}} \neq 0$, we get $w_{l_{1}}^{+}=w_{l_{1}}^{-}$. This holds for any $l_{1} \in N \backslash I_{\Phi^{+}, \Phi^{-}}$. Hence (11) becomes

$$
\forall s, s^{\prime} \in \Gamma \quad\left\{\begin{array}{l}
\sum_{l \in I_{\Phi^{+}, \Phi^{-}}}\left(w_{l}^{+}-w_{l}^{-}\right) \Delta s_{l}=0 \\
\sum_{l \in I_{\Phi^{+}, \Phi^{-}}} \alpha_{l} \Delta s_{l}=0
\end{array}\right.
$$

Let $l_{1}, l_{2} \in I_{\Phi^{+}, \Phi^{-}}$. There exists $s, s^{\prime} \in \Gamma$ such that $s_{l}^{\prime}=s_{l}$ if $l \neq l_{1}, l_{2}$, $\Delta s_{l_{1}} \neq 0$ and $\Delta s_{l_{2}} \neq 0$. Hence

$$
\left\{\begin{array}{l}
\left(w_{l_{1}}^{+}-w_{l_{1}}^{-}\right) \Delta s_{l_{1}}+\left(w_{l_{2}}^{+}-w_{l_{2}}^{-}\right) \Delta s_{l_{2}}=0 \\
\alpha_{l_{1}} \Delta s_{l_{1}}+\alpha_{l_{2}} \Delta s_{l_{2}}=0
\end{array}\right.
$$

This linear system in $\Delta s_{l_{1}}$ and $\Delta s_{l_{2}}$ has non trivial solutions if and only if

$$
\frac{w_{l_{1}}^{+}-w_{l_{1}}^{-}}{\alpha_{l_{1}}}=\frac{w_{l_{2}}^{+}-w_{l_{2}}^{-}}{\alpha_{l_{2}}} .
$$

Since this holds for all $l_{1}, l_{2} \in I_{\Phi^{+}, \Phi^{-}}$, there exists $\theta \in \mathbb{R}$ such that

$$
w_{l}^{+}-w_{l}^{-}=\theta \alpha_{l} \quad \forall l \in I_{\Phi^{+}, \Phi^{-}} .
$$

Since $\alpha_{l}=0$ for $l \notin I_{\Phi^{+}, \Phi^{-}}$, we get

$$
\exists \theta \in \mathbb{R} \quad \forall l \in N, \quad w_{l}^{+}-w_{l}^{-}=\theta \alpha_{l} .
$$

- Assume by contradiction that there exists an aggregation function $F$ that is continuous, piecewise linear for which (S2') is satisfied when criterion $i$ is the closest to the neutral level among criteria $i, j^{+}, j^{-}$. The first part of (S2') implies that $w_{j^{+}}(s)>w_{j^{-}}(s)$ when $s_{i}>0$ and 
the second part of $\left(\mathbf{S 2}^{\prime}\right)$ implies that $w_{j^{+}}(s)<w_{j^{-}}(s)$ when $s_{i}<0$. For some fixed values of $s_{-i}$, the weights of $F$ is conditional on the sign of $s_{i}$ for $\left|s_{i}\right|$ small enough. Hence, there exists two domains $\Phi^{+}$ and $\Phi^{-}$of $\Phi(F)$ such that $\Gamma:=\overline{\Phi^{+}} \cap \overline{\Phi^{-}}$is described by $s_{i}=0$ with $\Phi^{+} \subseteq\left\{s \in\right.$ reel $\left.^{n}, s_{i}>0\right\}, \Phi^{-} \subseteq\left\{s \in \mathbb{R}^{n}, s_{i}<0\right\}$, and

$$
\left\{\begin{array}{l}
\text { If } s \in \Phi^{+} \text {then } w_{j^{+}}^{+}>w_{j^{-}}^{+} \\
\text {If } s \in \Phi^{-} \text {then } w_{j^{+}}^{-}<w_{j^{-}}^{-}
\end{array}\right.
$$

Since $I_{\Phi^{+}, \Phi^{-}}=\{i\}$, relation (12) gives

$$
w_{l}^{+}=w_{l}^{-} \quad \forall l \neq i
$$

which contradicts previous relations.

Corollary 1 If criterion $i$ is the closest to the neutral level among criteria $i, j^{+}, j^{-}$, then the weights of criteria $j^{-}$and $j^{+}$are not conditional on the fact that criterion $i$ is attractive or repulsive.

Proof : Clear from the proof of Theorem 1.

The result of Theorem 1 is not true when $F$ is not piecewise linear. Consider indeed the following nonlinear aggregation function

$$
F(s)=\frac{1+s_{i}}{2} \times s_{j^{+}}+\frac{1}{2} \times s_{j^{-}} .
$$

Then the weight of criterion $j^{+}$is $\frac{\partial F}{\partial s_{j}+}(s)=\frac{1+s_{i}}{2}$ and that of criterion $j^{-}$is $\frac{\partial F}{\partial s_{j^{-}}}(s)=\frac{1}{2}$. Hence, statement (S2') is perfectly satisfied by $F$.

Theorem 1 can be interpreted in the following way. This result states that when $x_{i}$ is close to the neutral level $\mathbf{0}_{i}$ (relatively to criteria $j^{+}$and $j^{-}$), the relative preference of the actor over criteria $j^{+}$and $j^{-}$is not so clear. This is a hesitation area.

Let us show as an example that bi-capacities satisfy to the restriction imposed by previous lemma. By (4), when the scores w.r.t. criteria are all different in absolute value, the weight of criterion $l$ for an act $s \in \Omega$ for a bi-capacity $\mu$ is given by

$$
w_{l}(s)= \begin{cases}\mu\left(\{l\} \cup C_{l}^{+}(s), C_{l}^{-}(s)\right)-\mu\left(C_{l}^{+}(s), C_{l}^{-}(s)\right) & \text { if } s_{l} \geq 0 \\ \mu\left(C_{l}^{+}(s), C_{l}^{-}(s)\right)-\mu\left(C_{l}^{+}(s),\{l\} \cup C_{l}^{-}(s)\right) & \text { if } s_{l}<0\end{cases}
$$


where

$$
C_{l}^{+}(s)=\left\{m \neq l, s_{m} \geq 0 \text { and }\left|s_{m}\right| \geq\left|s_{l}\right|\right\}
$$

and

$$
C_{l}^{-}(s)=\left\{m \neq l, s_{m}<0 \text { and }\left|s_{m}\right| \geq\left|s_{l}\right|\right\} .
$$

If $\left|s_{i}\right|<\left|s_{l}\right|$ then $i \notin C_{l}^{+}(s) \cup C_{l}^{-}(s)$. Hence, if criterion $i$ is the closest to the neutral level among criteria $i, j^{+}, j^{-}$, then $i \notin C_{j^{+}}^{+}(s) \cup C_{j^{+}}^{-}(s)$ and $i \notin C_{j^{-}}^{+}(s) \cup C_{j^{-}}^{-}(s)$. This means that $w_{j^{+}}(s)$ and $w_{j^{-}}(s)$ do not depend on whether $s_{i} \geq 0$. Henceforth, (S2') cannot be modeled in this case. Now when $\left|s_{i}\right| \geq\left|s_{l}\right|$, then $i \in C_{l}^{+}(s)$ if $s_{i} \geq 0$ and $i \in C_{l}^{-}(s)$ if $s_{i}<0$. Hence, the weight $w_{l}(s)$ can can change between the two cases $s_{i} \geq 0$ and $s_{i}<0$. As a consequence, one cannot model (S2') with a bi-capacity whenever criterion $i$ is the closest to the neutral level among criteria $i, j^{+}, j^{-}$.

We restrict (S2') according to Theorem 1:

(S3): If the value w.r.t. criterion $i$ is attractive $(>0)$, and $i$ is not the closest to the neutral level among criteria $i, j^{+}, j^{-}$, then criterion $j^{+}$is more important than criterion $j^{-}$. If the value w.r.t. criterion $i$ is repulsive $(<0)$, and $i$ is not the closest to the neutral level among criteria $i, j^{+}, j^{-}$, then criterion $j^{+}$is less important than criterion $j^{-}$.

Let us give the requirements on a bi-capacity imposed by previous statement (S3). Define for a bi-capacity $\mu$ the operation $\Delta_{i}^{ \pm}$by

$$
\begin{aligned}
& \Delta_{i}^{+} \mu(A, B)=\mu(A \cup\{i\}, B)-\mu(A, B) \\
& \Delta_{i}^{-} \mu(A, B)=\mu(A, B)-\mu(A, B \cup\{i\})
\end{aligned}
$$

Lemma $2 A$ bi-capacity $\mu$ satisfies (S3) if and only if we have

$$
\Delta_{j^{+}}^{\epsilon_{j^{+}}} \mu(A, B)>\Delta_{j^{-}}^{\epsilon_{j^{-}}} \mu\left(A^{\prime}, B^{\prime}\right) \quad \forall\left(A, B, A^{\prime}, B^{\prime}\right) \in \Sigma_{i}^{+} \forall \epsilon_{j^{+}}, \epsilon_{j^{-}} \in\{-,+\}
$$

and

$$
\Delta_{j^{+}}^{\epsilon_{j+}} \mu(A, B)<\Delta_{j^{-}}^{\epsilon_{j^{-}}} \mu\left(A^{\prime}, B^{\prime}\right) \quad \forall\left(A, B, A^{\prime}, B^{\prime}\right) \in \Sigma_{i}^{-} \forall \epsilon_{j^{+}}, \epsilon_{j^{-}} \in\{-,+\}
$$

where

$$
\begin{aligned}
& \Sigma_{i}^{+}:=\left\{(A, B),\left(A^{\prime}, B^{\prime}\right) \in \mathcal{Q}\left(N \backslash\left\{j^{+}, j^{-}\right\}\right), i \in A \cup A^{\prime}\right. \\
&\text { and either } \left.(A, B) \sqsubseteq^{*}\left(A^{\prime}, B^{\prime}\right) \text { or }\left(A^{\prime}, B^{\prime}\right) \sqsubseteq^{*}(A, B)\right\} \\
& \Sigma_{i}^{-}:=\quad\left\{(A, B),\left(A^{\prime}, B^{\prime}\right) \in \mathcal{Q}\left(N \backslash\left\{j^{+}, j^{-}\right\}\right), i \in B \cup B^{\prime}\right. \\
&\left.\quad \text { and either }(A, B) \sqsubseteq^{*}\left(A^{\prime}, B^{\prime}\right) \text { or }\left(A^{\prime}, B^{\prime}\right) \sqsubseteq^{*}(A, B)\right\}
\end{aligned}
$$

and $(A, B) \sqsubseteq^{*}\left(A^{\prime}, B^{\prime}\right)$ if $A \subseteq A^{\prime}$ and $B \subseteq B^{\prime}$. Moreover, there exists at least one bi-capacity satisfying to all previous relations. 
Proof : Consider for example the first part of (S3). One has $w_{l}(s)=$ $\Delta_{l}^{\epsilon_{l}} \mu\left(C_{l}^{+}(s), C_{l}^{-}(s)\right)$ where $\epsilon_{l}$ is the sign of $s_{l}$. Hence the first part of (S3) is equivalent to

$$
\Delta_{j^{+}}^{\epsilon_{j+}} \mu\left(C_{j^{+}}^{+}(s), C_{j^{+}}^{-}(s)\right)>\Delta_{j^{-}}^{\epsilon_{j^{-}}} \mu\left(C_{j^{-}}^{+}(s), C_{j^{-}}^{-}(s)\right) .
$$

If $\left|s_{j^{+}}\right|>\left|s_{j^{-}}\right|$then $\left(C_{j^{+}}^{+}(s), C_{j^{+}}^{-}(s)\right) \sqsubseteq^{*}\left(C_{j^{-}}^{+}(s), C_{j^{-}}^{-}(s)\right)$. In the other case, we have $\left(C_{j^{-}}^{+}(s), C_{j^{-}}^{-}(s)\right) \bigsqcup^{*}\left(C_{j^{+}}^{+}(s), C_{j^{+}}^{-}(s)\right)$. Finally, since $i$ is not the closest to the neutral level among criteria $i, j^{+}, j^{-}$, then $i \in C_{j^{+}}^{+}(s) \cup C_{j^{+}}^{-}(s) \cup$ $C_{j^{-}}^{+}(s) \cup C_{j^{-}}^{-}(s)$. Moreover, since $s_{i}>0, i$ appears in the positive part: $i \in C_{j^{+}}^{+}(s) \cup C_{j^{-}}^{+}(s)$. We conclude that $\left(C_{j^{+}}^{+}(s), C_{j^{+}}^{-}(s), C_{j^{-}}^{+}(s), C_{j^{-}}^{-}(s)\right) \in \Sigma_{i}^{+}$. The second part of (S3) is dealt with similarly.

To show that the constraints of the bi-capacity are not contradictory, let us construct a bi-capacity satisfying to all constraints. It is easy to see that if bi-capacity $\mu$ satisfies for all $(A, B) \in \mathcal{Q}\left(N \backslash\left\{i, j^{+}, j^{-}\right\}\right)$and for all $\varepsilon \in\{+,-\}$,

$$
\Delta_{j^{-}}^{\varepsilon} \mu(A, B)=\left\{\begin{array}{l}
0 \text { if } i \in A \\
\alpha \text { if } i \in B \\
\alpha / 2 \text { if } i \notin A \cup B
\end{array}, \Delta_{j^{+}}^{\varepsilon} \mu(A, B)=\left\{\begin{array}{l}
\alpha \text { if } i \in A \\
0 \text { if } i \in B \\
\alpha / 2 \text { if } i \notin A \cup B
\end{array}\right.\right.
$$

where $\alpha>0$, then $\mu$ fulfills all constraints in Lemma 2 . It remains to construct a bi-capacity satisfying (13). This bi-capacity is independent of criteria $N \backslash\left\{i, j^{+}, j^{-}\right\}$. Let $(A, B) \in \mathcal{Q}\left(N \backslash\left\{i, j^{+}, j^{-}\right\}\right)$.

We set $\mu(A, B)=0$. From relation (13), we get

$$
\begin{array}{ll}
\mu\left(A \cup\left\{j^{-}\right\}, B\right)=\alpha / 2 & \mu\left(A \cup\left\{j^{+}\right\}, B\right)=\alpha / 2 \\
\mu\left(A, B \cup\left\{j^{-}\right\}\right)=-\alpha / 2 & \mu\left(A, B \cup\left\{j^{+}\right\}\right)=-\alpha / 2 \\
\mu\left(A \cup\left\{j^{-}\right\}, B \cup\left\{j^{+}\right\}\right)=0 & \mu\left(A \cup\left\{j^{+}\right\}, B \cup\left\{j^{-}\right\}\right)=0 \\
\mu\left(A, B \cup\left\{j^{-}, j^{+}\right\}\right)=-\alpha & \mu\left(A \cup\left\{j^{-}, j^{+}\right\}, B\right)=\alpha \\
\mu(A, B)=0 &
\end{array}
$$

We set $\mu\left(A \cup\left\{i, j^{+}, j^{-}\right\}, B\right)=1$. From relation (13), we get

$$
\begin{array}{ll}
\mu\left(A \cup\left\{i, j^{-}\right\}, B\right)=1-\alpha & \mu\left(A \cup\left\{i, j^{+}\right\}, B\right)=1 \\
\mu\left(A \cup\{i\}, B \cup\left\{j^{-}\right\}\right)=1-\alpha & \mu\left(A \cup\{i\}, B \cup\left\{j^{+}\right\}\right)=1-2 \alpha \\
\mu\left(A \cup\left\{i, j^{-}\right\}, B \cup\left\{j^{+}\right\}\right)=1-2 \alpha & \mu\left(A \cup\left\{i, j^{+}\right\}, B \cup\left\{j^{-}\right\}\right)=1 \\
\mu\left(A \cup\{i\}, B \cup\left\{j^{+}, j^{-}\right\}\right)=1-2 \alpha & \mu\left(A \cup\left\{i, j^{+}, j^{-}\right\}, B\right)=1 \\
\mu(A \cup\{i\}, B)=1-\alpha &
\end{array}
$$


We set $\mu\left(A, B \cup\left\{i, j^{+}, j^{-}\right\}\right)=-1$. From relation (13), we get

$$
\begin{array}{ll}
\mu\left(A \cup\left\{j^{-}\right\}, B \cup\{i\}\right)=2 \alpha-1 & \mu\left(A \cup\left\{j^{+}\right\}, B \cup\{i\}\right)=\alpha-1 \\
\mu\left(A, B \cup\left\{i, j^{-}\right\}\right)=-1 & \mu\left(A, B \cup\left\{i, j^{+}\right\}\right)=\alpha-1 \\
\mu\left(A \cup\left\{j^{-}\right\}, B \cup\left\{i, j^{+}\right\}\right)=2 \alpha-1 & \mu\left(A \cup\left\{j^{+}\right\}, B \cup\left\{i, j^{-}\right\}\right)=-1 \\
\mu\left(A, B \cup\left\{i, j^{+}, j^{-}\right\}\right)=-1 & \mu\left(A \cup\left\{j^{-}, j^{+}\right\}, B \cup\{i\}\right)=2 \alpha-1 \\
\mu(A, B \cup\{i\})=\alpha-1 &
\end{array}
$$

Clearly, $\mu$ is non-decreasing w.r.t. criteria of $N \backslash\{i\}$. Finally, $\mu$ is nondecreasing w.r.t. criterion $i$ iff $0 \leq \alpha \leq 1 / 2$.

\section{Determination of the neutral level}

We consider two types of attributes. An attribute $X_{k}$ can be cardinal (in this case $X_{k}$ is identified to a closed interval of $\mathbb{R}$ ) or ordinal (in this case $X_{k}$ is identified to a set of discrete items $\left\{x_{k}^{1}, \ldots, x_{k}^{p_{k}}\right\}$ ). For the sake of simplicity, we assume that if $X_{k}$ is cardinal, $x_{k} \geq x_{k}^{\prime}$ implies that $u_{k}\left(x_{k}\right) \geq u_{k}\left(x_{k}^{\prime}\right)$. Similarly, if $X_{k}$ is ordinal, $l \geq l^{\prime}$ implies that $u_{k}\left(x_{k}^{l}\right) \geq u_{k}\left(x_{k}^{l^{\prime}}\right)$. Our study can be easily carried over to the case when $u_{k}$ is not non-decreasing.

\subsection{General construction}

We have assumed so far the existence of three reference levels $-\mathbf{1}_{k}, \mathbf{0}_{k}$ and $\mathbf{1}_{k}$ on each attribute $X_{k}$ in Section 3.1. However, their determination may not be so obvious in practice. The two reference elements $-\mathbf{1}_{k}$ and $\mathbf{1}_{k}$ correspond to aspiration levels or anchors and their existence is justified by the theory of satisficing bounded rationality [21]. On the other hand, the neutral element $\mathbf{0}_{k}$ does not have such a strong anchor. We argue that the determination of such neutral elements is not a trivial matter. It is not easy for the actor to determine the precise boundary between attractiveness and repulsiveness since this latter is exogenous. The actor can easily give the elements of $X_{k}$ denoted by $X_{k}^{++}$that are clearly attractive, and the elements of $X_{k}$ denoted by $X_{k}^{--}$that are clearly repulsive. These are the elements of $X_{i}$ that are referred to in statement (S1). The neutral element $\mathbf{0}_{k}$ belongs to the set $X_{k} \backslash\left(X_{k}^{++} \cup X_{k}^{--}\right)$between the attractive elements and the repulsive ones, but the actor cannot say more.

As argued in [18], the unipolar or bipolar nature of a scale is not so clear in MCDA, which may explains why the determination of the neutral elements is not so obvious. We claim that a bipolar scale is required when decision behaviors are conditional on one or several attributes being attractive (i.e. 
good) or repulsive (i.e. bad). Statement (S2) is a typical example of this type of behavior. This means that the overall preference relation $\succeq$ exhibits behavioral switches between attractive and repulsive values. Thanks to an appropriate questionnaire, it may be possible to determine the transitions between typical behaviors for attractive values and typical behaviors for repulsive values, and deduce from that an estimate of the value of the neutral element. We will make this study in the case of Statement (S3).

We shall first construct the utility functions without using the unknown neutral elements. Hence, unlike what was done in Section 3.1, the positive and the negative parts of the utility functions cannot be constructed separately. For the Choquet integral w.r.t. a bi-capacity, it is not possible to find $z_{-k}$ satisfying (5). This means that whatever the choice of $z_{-k}$, the set $\left\{\left(u_{k}\left(x_{k}\right), u_{-k}\left(z_{-k}\right)\right), x_{k} \in X_{k}\right\}$ is included in the union of at least two domains of $\Phi\left(\mathcal{B C}_{\mu}\right)$. Hence, criterion $k$ in $U\left(x_{k}, z_{-k}\right)$ probably has at least two different weights when $x_{k}$ sweeps $X_{k}$. Hence, the perception of $u_{k}$ may be altered by $U$.

We use other reference levels than the ones defined in Section 3.1. It is assumed that there exists an element denoted by $\mathbf{U}_{k}$ which is considered as completely unsatisfactory for the actor, and an element denoted by $\mathbf{P}_{k}$ that is considered as perfectly satisfactory on point of view $X_{k}$ [17]. We propose two options for the choice of $z_{-k}$ : either $\mathbf{P}_{-k}$ or $\mathbf{U}_{-k}$. We define $u_{k}^{\star}$ such that $x_{k} \mapsto u_{k}^{\star}\left(x_{k}\right)$ is an equivalent interval scale to $x_{k} \mapsto U\left(x_{k}, \mathbf{P}_{-k}\right)$ (resp. $\left.U\left(x_{k}, \mathbf{U}_{-k}\right)\right)$ such that $u_{k}^{\star}\left(\mathbf{U}_{k}\right)=0, u_{k}^{\star}\left(\mathbf{P}_{k}\right)=1$ :

$u_{k}^{\star}\left(x_{k}\right)=\frac{U\left(x_{k}, \mathbf{P}_{-k}\right)-U\left(\mathbf{U}_{k}, \mathbf{P}_{-k}\right)}{U\left(\mathbf{P}_{N}\right)-U\left(\mathbf{U}_{k}, \mathbf{P}_{-k}\right)}\left(\operatorname{resp} . u_{k}^{\star}\left(x_{k}\right)=\frac{U\left(x_{k}, \mathbf{U}_{-k}\right)-U\left(\mathbf{U}_{N}\right)}{U\left(\mathbf{P}_{k}, \mathbf{U}_{-k}\right)-U\left(\mathbf{U}_{N}\right)}\right)$

The presence of the neutral element $\mathbf{0}_{k}$ between $\mathbf{U}_{k}$ and $\mathbf{P}_{k}$ may alter the perception of $u_{k}$ through $U\left(x_{k}, \mathbf{U}_{-k}\right)$ (resp. $U\left(x_{k}, \mathbf{P}_{-k}\right)$ ). Hence, $u_{k}^{\star}$ may differ from $u_{k}$. One can make an analogy with the perception of distances above or below water surface. The neutral element is here associated to the water surface. If the operator is above the water surface, his perception of distances is correct for objects above the water surface but is altered by the water diffraction for objects under the water level. Our problem is somehow similar to that problem in which the water is so crystal clear that the operator cannot identify the location of the water surface.

Even-though (5) cannot be satisfied, we make the assumption that for all $k \neq i$, the perception of $u_{k}$ is not altered by $U$. Hence, we have for all $k \neq i$ $\forall x_{k} \in X_{k}, U\left(x_{k}, \mathbf{P}_{-k}\right)=a_{k}^{\prime} u_{k}\left(x_{k}\right)+b_{k}^{\prime}\left(\operatorname{resp} . U\left(x_{k}, \mathbf{U}_{-k}\right)=a_{k}^{\prime} u_{k}\left(x_{k}\right)+b_{k}^{\prime}\right)$ 
that is for all $s_{k} \in\left[u_{k}\left(\mathbf{U}_{k}\right), u_{k}\left(\mathbf{P}_{k}\right)\right]$

$$
F\left(s_{k}, u_{-k}\left(\mathbf{P}_{-k}\right)\right)=a_{k}^{\prime} s_{k}+b_{k}^{\prime} \quad\left(\operatorname{resp} . F\left(s_{k}, u_{-k}\left(\mathbf{U}_{-k}\right)\right)=a_{k}^{\prime} s_{k}+b_{k}^{\prime}\right) .
$$

Combining (14) and (15), we get

$$
\forall x_{k} \in X_{k}, \quad u_{k}\left(x_{k}\right)=\alpha_{k} u_{k}^{\star}\left(x_{k}\right)+\beta_{k} .
$$

Let us now determine the values of $\alpha_{k}$ and $\beta_{k} . u_{k}$ is thus constructed from elements $\mathbf{0}_{k}, \mathbf{U}_{k}$ and $\mathbf{P}_{k}$ instead of $\mathbf{0}_{k},-\mathbf{1}_{k}$ and $\mathbf{1}_{k}$. One wish still to have $u_{k}\left(\mathbf{0}_{k}\right)=0$. However, it does not make sense to enforce that $u_{k}\left(\mathbf{P}_{k}\right)=1$ and $u_{k}\left(\mathbf{U}_{k}\right)=-1$ since the neutral element $\mathbf{0}_{k}$ is not necessarily put right in the middle of elements $\mathbf{U}_{k}$ and $\mathbf{P}_{k}$. Moreover, one needs only one more equality to determine the values of $\alpha_{k}$ and $\beta_{k}$. We enforce $u_{k}\left(\mathbf{P}_{k}\right)-u_{k}\left(\mathbf{U}_{k}\right)=$ $u_{k}^{\star}\left(\mathbf{P}_{k}\right)-u_{k}^{\star}\left(\mathbf{U}_{k}\right)=1$. We conclude that $\alpha_{k}=1$ and $\beta_{k}=-u_{k}^{\star}\left(\mathbf{0}_{k}\right)$ :

$$
u_{k}\left(x_{k}\right)=u_{k}^{\star}\left(x_{k}\right)-u_{k}^{\star}\left(\mathbf{0}_{k}\right) \text {. }
$$

Since the $u_{k}^{\star}$ scales are commensurate for $k \neq i$, and the $\mathbf{0}_{k}$ levels have the same meaning, one has

$$
u_{1}^{\star}\left(\mathbf{0}_{1}\right)=\cdots=u_{i-1}^{\star}\left(\mathbf{0}_{i-1}\right)=u_{i+1}^{\star}\left(\mathbf{0}_{i+1}\right)=\cdots=u_{n}^{\star}\left(\mathbf{0}_{n}\right)=: t .
$$

Value $t$ is unknown and has to be determined. Hence (see Fig. 1)

$$
u_{k}\left(x_{k}\right)=u_{k}^{\star}\left(x_{k}\right)-t
$$

Due to (S3), attribute $i$ is clearly of bipolar nature so that the presence of the neutral element $\mathbf{0}_{i}$ certainly alters the perception of $u_{i}$ through $U\left(x_{i}, \mathbf{U}_{-i}\right)$ (resp. $\left.U\left(x_{i}, \mathbf{P}_{-i}\right)\right)$. Hence, (see (6))

$$
\left\{\begin{array}{l}
\forall x_{i} \succeq_{i} \mathbf{0}_{i}, \quad U\left(x_{i}, \mathbf{P}_{-i}\right)=a_{i}^{\prime} u_{i}\left(x_{i}\right)+b_{i}^{\prime} \quad\left(\operatorname{resp.} U\left(x_{i}, \mathbf{U}_{-i}\right)=a_{i}^{\prime} u_{i}\left(x_{i}\right)+b_{i}^{\prime}\right) \\
\forall x_{i} \preceq_{i} \mathbf{0}_{i}, \quad U\left(x_{i}, \mathbf{P}_{-i}\right)=a_{i}^{\prime \prime} u_{i}\left(x_{i}\right)+b_{i}^{\prime \prime} \quad\left(\operatorname{resp} . U\left(x_{i}, \mathbf{U}_{-i}\right)=a_{i}^{\prime \prime} u_{i}\left(x_{i}\right)+b_{i}^{\prime \prime}\right)
\end{array}\right.
$$

By (14)

$$
\begin{cases}\forall x_{i} \succeq_{i} \mathbf{0}_{i}, & u_{i}\left(x_{i}\right)=\alpha_{i}^{+} u_{i}^{\star}\left(x_{i}\right)+\beta_{i}^{+} \\ \forall x_{i} \preceq_{i} \mathbf{0}_{i}, & u_{i}\left(x_{i}\right)=\alpha_{i}^{-} u_{i}^{\star}\left(x_{i}\right)+\beta_{i}^{-}\end{cases}
$$

By continuity of function $u_{i}$, adding constraints $u_{i}\left(\mathbf{P}_{i}\right)-u_{i}\left(\mathbf{U}_{i}\right)=1$ and $u_{i}\left(\mathbf{0}_{i}\right)=0$, we have

$$
u_{i}\left(x_{i}\right)=T\left(u_{i}^{\star}\left(x_{i}\right)\right)
$$

where $T$ is piecewise affine and is characterized by the three points $x_{i}=\mathbf{U}_{i}$, $x_{i}=\mathbf{0}_{i}$ and $x_{i}=\mathbf{P}_{i}$. By commensurateness of the $\mathbf{U}_{k}$ levels and (16), one 
has $T(0)=T\left(u_{i}^{\star}\left(\mathbf{U}_{i}\right)\right)=u_{i}\left(\mathbf{U}_{i}\right)=u_{k}\left(\mathbf{U}_{k}\right)=-t$. By commensurateness of the $\mathbf{P}_{k}$ levels and (16), one has $T(1)=T\left(u_{i}^{\star}\left(\mathbf{P}_{i}\right)\right)=u_{i}\left(\mathbf{P}_{i}\right)=u_{k}\left(\mathbf{P}_{k}\right)=1-t$. Moreover, $0=u_{i}\left(\mathbf{0}_{i}\right)=T\left(u_{i}^{\star}\left(\mathbf{0}_{i}\right)\right)$. The perception of $\mathbf{0}_{i}$ through the unipolar assumption is not the same as that with the bipolar assumption. Hence $\mathbf{0}_{i}$ is not similar to $\mathbf{0}_{k}$ in the unipolar view: $u_{i}^{\star}\left(\mathbf{0}_{i}\right) \neq t$. We set $b=u_{i}^{\star}\left(\mathbf{0}_{i}\right)$. Hence $T(b)=0$. This gives (see Fig. 1)

$$
T(\theta)= \begin{cases}\frac{(1-t)(\theta-b)}{1-b} & \text { if } \theta \geq b \\ \frac{t(\theta-b)}{b} & \text { if } \theta<b\end{cases}
$$

Values $t$ and $b$ are unknown.
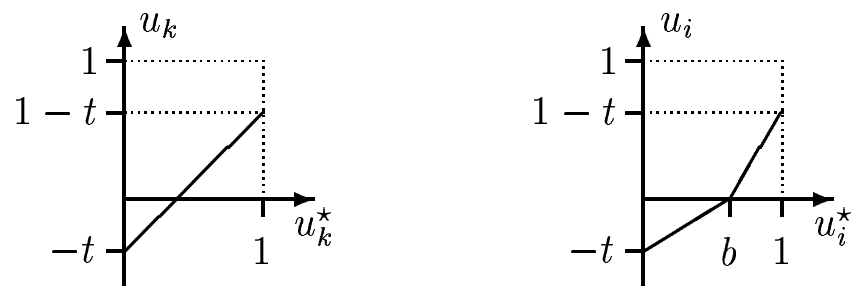

Figure 1: Functions $u_{k}^{\star} \mapsto u_{k}(k \neq i)$ and $u_{i}^{\star} \mapsto u_{i}$.

In Section 4.2, we aim at determining both $t$ and $b$ from a questionnaire.

When assumption (15) holds for all $k \in N$, the construction of the utility functions $u_{k}$ from levels $\mathbf{U}$ and $\mathbf{P}$ is not altered by the aggregation function $F$. This implies that $b=t$ in Figure 1. We describe in Section 4.3 a method for determining the value of $t$ under this assumption.

\subsection{Determination of the neutral element and a renor- malization of criterion $i$}

The following question is asked to the actor according to statement (S3).

Question Q1 $(x)$. Considering option $x$, if the value of $x$ could be improved on either criterion $j^{+}$or criterion $j^{-}$, which criteria would you prefer?

- " $j^{+} \succ j^{-}$": I prefer to improve criterion $j^{+}$.

- " $j^{+} \prec j^{-}$": I prefer to improve criterion $j^{-}$.

- " $j^{+} \sim j^{-}$": I have no preference between $j^{+}$and $j^{-}$.

Lemma 3 Consider an option $x$ such that $x_{k}=\mathbf{P}_{k}$ (resp. $x_{k}=\mathbf{U}_{k}$ ) for $k \in N \backslash\left\{i, j^{+}, j^{-}\right\}$. For $x_{-i}$ fixed, we ask question Q1 $(x)$ for "all" $x_{i} \in$ 
$X_{i}$. Assume that the actor satisfies to statement (S3). Then if there exists $x_{i} \in X_{i}$ with $\left|u_{i}\left(x_{i}\right)\right|<\min \left(\left|u_{j^{+}}\left(x_{j^{+}}\right)\right|,\left|u_{j^{-}}\left(x_{j^{-}}\right)\right|\right)$, such that the answer to Q1(x) is " $j^{+} \succ j^{-}$", then (see Fig. 2.a)

- For all $x_{i} \in X_{i}$, with $u_{i}\left(x_{i}\right)>-\min \left(\left|u_{j^{+}}\left(x_{j^{+}}\right)\right|,\left|u_{j^{-}}\left(x_{j^{-}}\right)\right|\right)$, the answer to Q1 $(x)$ shall be " $j^{+} \succ j^{-}$".

- For all $x_{i} \in X_{i}$, with $u_{i}\left(x_{i}\right)<-\min \left(\left|u_{j^{+}}\left(x_{j^{+}}\right)\right|,\left|u_{j^{-}}\left(x_{j^{-}}\right)\right|\right)$, the answer to Q1 $(x)$ shall be " $j^{+} \prec j^{-}$".

If there exists $x_{i} \in X_{i}$ with $\left|u_{i}\left(x_{i}\right)\right|<\min \left(\left|u_{j^{+}}\left(x_{j^{+}}\right)\right|,\left|u_{j^{-}}\left(x_{j^{-}}\right)\right|\right)$, such that the answer to Q1(x) is " $j^{+} \prec j^{-}$", then (see Fig. 2.b)

- For all $x_{i} \in X_{i}$, with $x_{i} \succ_{i} \mathbf{0}_{i}$ and $u_{i}\left(x_{i}\right)>\min \left(\left|u_{j^{+}}\left(x_{j^{+}}\right)\right|,\left|u_{j^{-}}\left(x_{j^{-}}\right)\right|\right)$, the answer to Q1 $(x)$ shall be " $j^{+} \succ j^{-}$".

- For all $x_{i} \in X_{i}$, with $x_{i} \prec_{i} \mathbf{0}_{i}$ and $u_{i}\left(x_{i}\right)<\min \left(\left|u_{j^{+}}\left(x_{j^{+}}\right)\right|,\left|u_{j^{-}}\left(x_{j^{-}}\right)\right|\right)$, the answer to Q1 $(x)$ shall be " $j^{+} \prec j^{-}$".

If there exists $x_{i} \in X_{i}$ with $\left|u_{i}\left(x_{i}\right)\right|<\min \left(\left|u_{j^{+}}\left(x_{j^{+}}\right)\right|,\left|u_{j^{-}}\left(x_{j^{-}}\right)\right|\right)$, such that the answer to $\mathbf{Q 1}(x)$ is " $j^{+} \sim j^{-}$", then (see Fig. 2.c)

- For all $x_{i} \in X_{i}$, with $x_{i} \succ_{i} \mathbf{0}_{i}$ and $u_{i}\left(x_{i}\right)>\min \left(\left|u_{j^{+}}\left(x_{j^{+}}\right)\right|,\left|u_{j^{-}}\left(x_{j^{-}}\right)\right|\right)$, the answer to Q1 $(x)$ shall be " $j^{+} \succ j^{-}$".

- For all $x_{i} \in X_{i}$ such that $\left|u_{i}\left(x_{i}\right)\right|<\min \left(\left|u_{j^{+}}\left(x_{j^{+}}\right)\right|,\left|u_{j^{-}}\left(x_{j^{-}}\right)\right|\right)$, the answer to Q1 $(x)$ is " $j^{+} \sim j^{-}$".

- For all $x_{i} \in X_{i}$, with $x_{i} \prec_{i} \mathbf{0}_{i}$ and $u_{i}\left(x_{i}\right)<-\min \left(\left|u_{j^{+}}\left(x_{j^{+}}\right)\right|,\left|u_{j^{-}}\left(x_{j^{-}}\right)\right|\right)$, the answer to Q1 $(x)$ shall be " $j^{+} \prec j^{-}$".

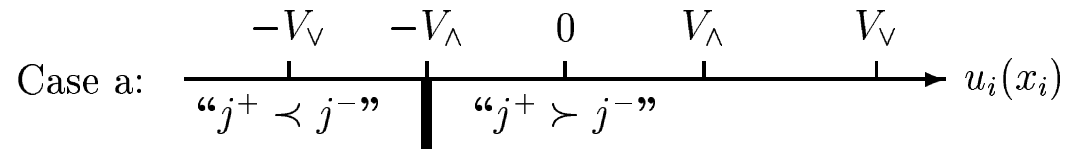

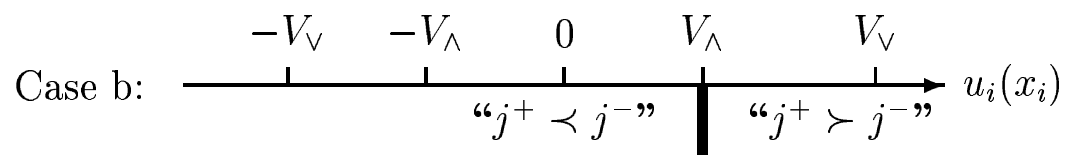

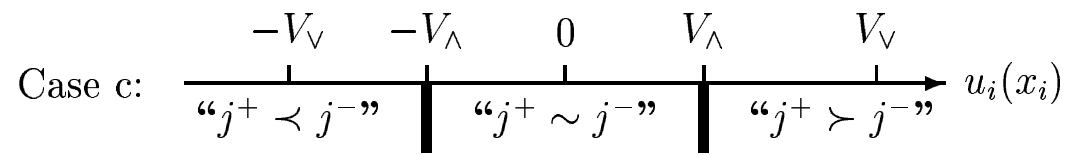

Figure 2: The three cases with the notations $V_{\wedge}=$ $\min \left(\left|u_{j^{+}}\left(x_{j^{+}}\right)\right|,\left|u_{j^{-}}\left(x_{j^{-}}\right)\right|\right), V_{\vee}=\max \left(\left|u_{j^{+}}\left(x_{j^{+}}\right)\right|,\left|u_{j^{-}}\left(x_{j^{-}}\right)\right|\right)$. 
Proof : For all $x_{i} \in X_{i}$, with $x_{i} \succ_{i} \mathbf{0}_{i}$ and $u_{i}\left(x_{i}\right)>\min \left(\left|u_{j^{+}}\left(x_{j^{+}}\right)\right|,\left|u_{j^{-}}\left(x_{j^{-}}\right)\right|\right)$, then by (S3), the answer to Q1 $(x)$ shall be " $j^{+} \succ j^{-}$". For all $x_{i} \in X_{i}$, with $x_{i} \prec_{i} \mathbf{0}_{i}$ and $u_{i}\left(x_{i}\right)<-\min \left(\left|u_{j^{+}}\left(x_{j^{+}}\right)\right|,\left|u_{j^{-}}\left(x_{j^{-}}\right)\right|\right)$, then by (S3), the answer to Q1 $(x)$ shall be " $j^{+} \prec j^{-}$". According to Corollary 1, the answer should be the same for all $x_{i} \in X_{i}$ such that $\left|u_{i}\left(x_{i}\right)\right|<\min \left(\left|u_{j^{+}}\left(x_{j^{+}}\right)\right|,\left|u_{j^{-}}\left(x_{j^{-}}\right)\right|\right)$.

When $x_{i}$ sweeps $X_{i}$ from $\mathbf{U}_{i}$ to $\mathbf{P}_{i}$, according to Lemma 3 , the number of changes of preferences between $j^{+}$and $j^{-}$is either one or two. These changes are also called transitions. One transition may appear for $x_{i}$ such that $u_{i}\left(x_{i}\right)=-\min \left(\left|u_{j^{+}}\left(x_{j^{+}}\right)\right|,\left|u_{j^{-}}\left(x_{j^{-}}\right)\right|\right)$, and the second one may appear for $x_{i}$ with $u_{i}\left(x_{i}\right)=\min \left(\left|u_{j^{+}}\left(x_{j^{+}}\right)\right|,\left|u_{j^{-}}\left(x_{j^{-}}\right)\right|\right)$.

For $x_{k}, x_{k}^{\prime} \in X_{k}$, with $x_{k} \neq x_{k}^{\prime}$ we define by $\mathcal{M}_{k}\left(x_{k}, x_{k}^{\prime}\right)$ the mean value of $x_{k}$ and $x_{k}^{\prime}$. More precisely, if $X_{k}$ is cardinal, $\mathcal{M}_{k}\left(x_{k}, x_{k}^{\prime}\right)=\frac{x_{k}+x_{k}^{\prime}}{2}$. If $X_{k}$ is ordinal, then $\mathcal{M}_{k}\left(x_{k}, x_{k}^{\prime}\right)=x_{k}^{\left\lfloor\left(l+l^{\prime}\right) / 2\right\rfloor}$ where $x_{k}=x_{k}^{l}, x_{k}^{\prime}=x_{k}^{l^{\prime}}$ and $\lfloor\alpha\rfloor$ denotes the closest integer value to $\alpha$. We define $d_{k}\left(x_{k}, x_{k}^{\prime}\right)$ as the distance between $x_{k}$ and $x_{k}^{\prime}$. If $X_{k}$ is cardinal, $d_{k}\left(x_{k}, x_{k}^{\prime}\right)=\left|x_{k}-x_{k}^{\prime}\right|$. If $X_{k}$ is ordinal, $d_{k}\left(x_{k}, x_{k}^{\prime}\right)=\left|l-l^{\prime}\right|$ where $x_{k}=x_{k}^{l}, x_{k}^{\prime}=x_{k}^{l^{\prime}}$. We define $\varepsilon_{k}=\varepsilon$ if $X_{k}$ is cardinal (with $\varepsilon \in(0,1)$ ), and $\varepsilon_{k}=2$ if $X_{k}$ is ordinal. Hence, if $X_{k}$ is ordinal, $d_{k}\left(x_{k}, x_{k}^{\prime}\right) \geq \varepsilon_{k}$ iff there exists at least one element of $X_{k}$ different from $x_{k}$ and $x_{k}^{\prime}$ that is strictly bracketed between $x_{k}$ and $x_{k}^{\prime}$.

We fix $x_{-i}$ such that for all $k \in N \backslash\left\{i, j^{+}, j^{-}\right\}, x_{k} \in\left\{\mathbf{U}_{k}, \mathbf{P}_{k}\right\}$. The transitions can be determined by dichotomy:

\section{Algorithm $\operatorname{Alg} 1\left(x_{-i}\right)$}

Step 1. Let $\underline{x_{i}}:=\mathbf{U}_{i}$ and $\overline{x_{i}}=\mathbf{P}_{i}$. The answer to $\mathbf{Q 1}\left(\underline{x_{i}}, x_{-i}\right)$ is " $j^{+} \prec j^{-}$", that to $\mathbf{Q 1}\left(\overline{x_{i}}, x_{-i}\right)$ is " $j^{+} \succ j^{-}$".

Step 2. Let $x_{i}=\mathcal{M}_{i}\left(\underline{x_{i}}, \overline{x_{i}}\right)$. We have three cases:

Step 2.1. If the answer to $\mathbf{Q} \mathbf{1}(x)$ is " $j^{+} \succ j^{-}$", then we set $\overline{x_{i}}=$ $x_{i}$. If $d_{i}\left(\overline{x_{i}}, x_{i}\right)<\varepsilon_{i}$, we stop the algorithm: there is only one transition and interval $\left(x_{i}, \overline{x_{i}}\right)$ is an estimate of its value. If $d_{i}\left(\overline{x_{i}}, x_{i}\right) \geq \varepsilon_{i}$, goto Step 2 .

Step 2.2. If the answer to $\mathbf{Q} \mathbf{1}(x)$ is " $j^{+} \prec j^{-}$", then we set $x_{i}=$ $x_{i}$. If $\overline{x_{i}}-x_{i}<\varepsilon$, we stop the algorithm: there is only one transition and interval $\left(\underline{x_{i}}, \overline{x_{i}}\right)$ is an estimate of its value. If $\overline{x_{i}}-\underline{x_{i}} \geq \varepsilon$, goto Step 2 .

Step 2.3. If the answer to $\mathbf{Q} \mathbf{1}(x)$ is " $j^{+} \sim j^{-}$", then there are two transitions. Set $\widehat{x_{i}}:=x_{i}$ and $\check{x_{i}}=x_{i}$ 
Step 2.4. Let $x_{i}=\mathcal{M}_{i}\left(x_{i}, \check{x}_{i}\right)$. We have two cases: If the answer to $\mathbf{Q 1}(x)$ is " $j^{+} \prec j^{-}$", then we set $\underline{x_{i}}=x_{i}$. Otherwise, the answer to $\mathbf{Q 1}(x)$ is " $j^{+} \sim j^{-}$", then we set $\check{x}_{i}=x_{i}$. If $d_{i}\left(\check{x}_{i}, x_{i}\right) \geq \varepsilon_{i}$, goto Step 2.4. Otherwise, interval $\left(\underline{x_{i}}, \check{x}_{i}\right)$ is an estimate of the value of the left transition.

Step 2.5. Let $x_{i}=\mathcal{M}_{i}\left(\overline{x_{i}}, \widehat{x_{i}}\right)$. We have two cases: If the answer to $\mathbf{Q} \mathbf{1}(x)$ is " $j^{+} \succ j^{-}$", then we set $\overline{x_{i}}=x_{i}$. Otherwise, the answer to $\mathbf{Q} \mathbf{1}(x)$ is " $j^{+} \sim j^{-}$", then we set $\widehat{x_{i}}=x_{i}$. If $d_{i}\left(\overline{x_{i}}, \widehat{x}_{i}\right) \geq \varepsilon_{i}$, goto Step 2.5. Otherwise, interval $\left(\overline{x_{i}}, \widehat{x_{i}}\right)$ is an estimate of the value of the right transition.

This method for estimating the value(s) of the transition(s) is close to the techniques developed in Decision Under Risk for assessing the certainty equivalent of a lottery $[9,15]$.

From the notations of Figure 2, there is a one-to-one mapping $M$

$$
M:\left\{-V_{\vee},-V_{\wedge}, V_{\wedge}, V_{\vee}\right\} \rightarrow\left\{u_{j^{+}}\left(x_{j^{+}}\right), u_{j^{-}}\left(x_{j^{-}}\right),-u_{j^{+}}\left(x_{j^{+}}\right),-u_{j^{-}}\left(x_{j^{-}}\right)\right\}
$$

However, without a knowledge of whether $x_{j^{+}} \succ_{j^{+}} \mathbf{0}_{j^{+}}$and $x_{j^{-}} \succ_{j^{-}} \mathbf{0}_{j^{-}}$, it is not possible to determine precisely $M$, that is the value of the potential transitions. So, we consider $x_{j^{+}} \in X_{j^{+}}^{++} \cup X_{j^{+}}^{--}$and $x_{j^{-}} \in X_{j^{-}}^{++} \cup X_{j^{-}}^{--}$so that the values of the two potential transitions $M\left(-V_{\wedge}\right)$ and $M\left(V_{\wedge}\right)$ are known. In Sections 4.2.1 and 4.2.3, we show that the values of $b$ and $t$ can be derived when there are two transitions. Yet, if there is only one transition, it is not possible to know beforehand whether it corresponds to $-V_{\wedge}$ or to $V_{\wedge}$. We propose in Section 4.2.2 and 4.2.4 to change slightly the value of $x_{j}$ and $x_{j^{-}}$ in a given direction and to investigate whether the resulting transition moves in the same direction. This enables to identify to which element $-V_{\wedge}$ or $V_{\wedge}$ the transition corresponds to. We sum up in Section 4.2.5 the cases where parameters $b$ and $t$ are fully determined. Surprisingly, the only case where $b$ and $t$ may not be fully specified is when $b=t$, that is when $\mathbf{0}_{i}$ does not alter the perception of $u_{i}$ through $U$. This case is studied in Section 4.3.

\subsubsection{First elements of $X^{++}$}

We start the questionnaire with $x_{j^{+}} \in X_{j^{+}}^{++}$and $x_{j^{-}} \in X_{j^{-}}^{++}$. Without loss of generality, we assume that $u_{j^{+}}^{\star}\left(x_{j^{+}}\right) \leq u_{j^{-}}^{\star}\left(x_{j^{-}}\right)$. Alg1 is applied on these values. One obtains either one single transition with a value in the estimated interval $\left(\underline{x_{i}}, \overline{x_{i}}\right)$, or two transitions with values in the estimated intervals $\left(\underline{x_{i}^{l}}, \overline{x_{i}^{l}}\right)$ and $\left(\underline{x_{i}^{r}}, \overline{x_{i}^{r}}\right)$ for the left and right transitions respectively. 


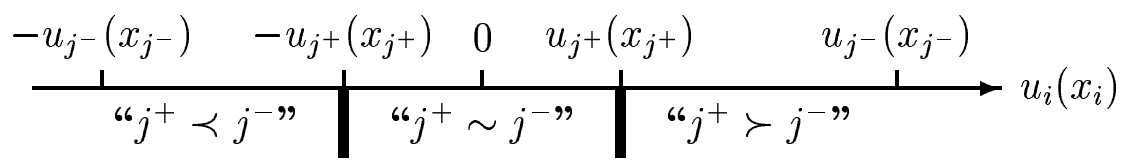

Figure 3: The two transitions.

If there is only one transition, we go to Section 4.2.2. We consider thus here the case of a double transition.

The exact left transition $x_{i}^{l}$ satisfies $u_{i}\left(x_{i}^{l}\right) \in\left(u_{i}\left(\underline{x_{i}^{l}}\right), u_{i}\left(\overline{x_{i}^{l}}\right)\right)$. From the third case of Lemma $3, u_{i}\left(x_{i}^{l}\right)=-u_{j^{+}}\left(x_{j^{+}}\right)$. Hence $-u_{j^{+}}\left(x_{j^{+}}\right) \in\left(u_{i}\left(\underline{x_{i}^{l}}\right), u_{i}\left(\overline{x_{i}^{l}}\right)\right)$. Since $\underline{x_{i}^{l}}$ and $\overline{x_{i}^{l}}$ are repulsive, one has $u_{i}\left(\underline{x_{i}^{l}}\right)=\frac{t}{b}\left(u_{i}^{\star}\left(\underline{x_{i}^{l}}\right)-b\right)$ and $u_{i}\left(\overline{x_{i}^{l}}\right)=$ $\frac{t}{b}\left(u_{i}^{\star}\left(\overline{x_{i}^{l}}\right)-b\right)$. Thus

$$
t-u_{j^{+}}^{\star}\left(x_{j^{+}}\right) \in\left(\frac{t}{b}\left(u_{i}^{\star}\left(\underline{x_{i}^{l}}\right)-b\right), \frac{t}{b}\left(u_{i}^{\star}\left(\overline{x_{i}^{l}}\right)-b\right)\right)
$$

The exact right transition $x_{i}^{r}$ satisfies $u_{i}\left(x_{i}^{r}\right) \in\left(u_{i}\left(\underline{x_{i}^{r}}\right), u_{i}\left(\overline{x_{i}^{r}}\right)\right)$. From the third case of Lemma $3, u_{i}\left(x_{i}^{r}\right)=u_{j^{+}}\left(x_{j^{+}}\right)$. Hence $u_{j^{+}}\left(x_{j^{+}}\right) \in\left(u_{i}\left(\underline{x_{i}^{r}}\right), u_{i}\left(\overline{x_{i}^{r}}\right)\right)$. Since $\underline{x_{i}^{r}}$ and $\overline{x_{i}^{r}}$ are attractive, one obtains

$$
\left.u_{j^{+}}^{\star}\left(x_{j^{+}}\right)-t \in\left(\frac{1-t}{1-b}\left(u_{i}^{\star} \underline{x_{i}^{r}}\right)-b\right), \frac{1-t}{1-b}\left(u_{i}^{\star}\left(\overline{x_{i}^{r}}\right)-b\right)\right)
$$

Consider first the case when there is no uncertainty (i.e. $\underline{x_{i}^{l}}=\overline{x_{i}^{l}}=x_{i}^{l}$ and $\left.\underline{x_{i}^{r}}=\overline{x_{i}^{r}}=x_{i}^{r}\right)$

$$
\left\{\begin{array}{l}
\frac{t}{b}\left(u_{i}^{\star}\left(x_{i}^{l}\right)-b\right)=t-u_{j^{+}}^{\star}\left(x_{j^{+}}\right) \\
\frac{1-t}{1-b}\left(u_{i}^{\star}\left(x_{i}^{r}\right)-b\right)=u_{j^{+}}^{\star}\left(x_{j^{+}}\right)-t
\end{array}\right.
$$

which gives

$$
\left\{\begin{array}{l}
t=\frac{u_{j^{+}}^{\star}\left(x_{j+}\right) \times b}{2 b-u_{i}^{\star}\left(x_{i}^{l}\right)}=: f_{1}(b) \\
t=\frac{\left(1-u_{j^{+}}^{\star}\left(x_{j+}\right)\right) \times b+u_{j+}^{\star}\left(x_{j+}\right)-u_{i}^{\star}\left(x_{i}^{r}\right)}{1-u_{i}^{\star}\left(x_{i}^{r}\right)}=: f_{2}(b)
\end{array}\right.
$$

Function $f_{2}$ is affine and increasing. Moreover $f_{2}(1)=1$. One has

$$
f_{1}^{\prime}(b)=-\frac{u_{j^{+}}^{\star}\left(x_{j^{+}}\right) \times u_{i}^{\star}\left(x_{i}^{l}\right)}{\left(2 b-u_{i}^{\star}\left(x_{i}^{l}\right)\right)^{2}}<0
$$

$f_{1}$ is an hyperbolic curve with the $x$-asymptote at value $\frac{u_{i}^{\star}\left(x_{i}^{l}\right)}{2} \in(0,1)$ and the $y$-asymptote at value $\frac{u_{j^{+}}^{\star}\left(x_{j+}\right)}{2} \in(0,1)$. Moreover, for $b=1, t=f_{1}(1)=$ 
$\frac{u_{j^{+}}^{\star}\left(x_{j^{+}}\right)}{2-u_{i}^{\star}\left(x_{i}^{l}\right)}$. This value is lower than 1 since $u_{j^{+}}^{\star}\left(x_{j^{+}}\right)+u_{i}^{\star}\left(x_{i}^{l}\right)<2$. Hence the upper right part of curve $f_{1}$ is "below" point $(b, t)=(1,1)$.

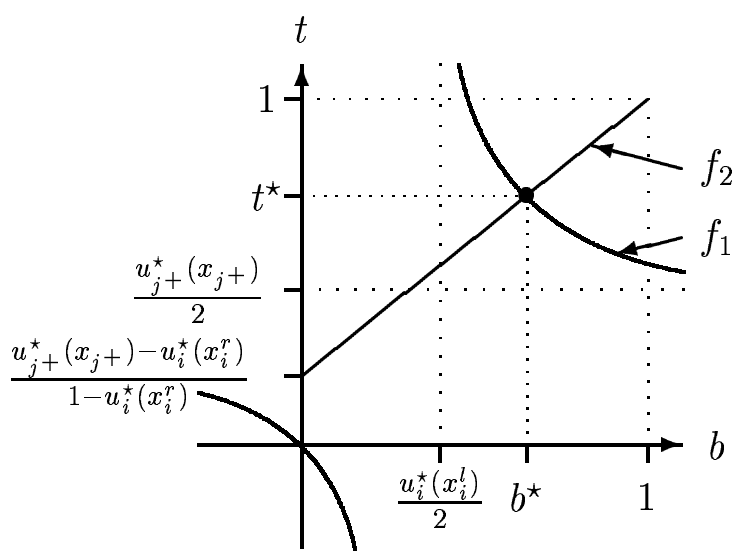

Figure 4: Functions $f_{1}$ and $f_{2}$.

We conclude that $f_{1}$ and $f_{2}$ intersect exactly at one point $\left(b^{\star}, t^{\star}\right) \in(0,1)^{2}$ (see Fig. 4). Hence there is a single solution to our problem.

When we take into account the uncertainties in $x_{i}^{l}$ and $x_{i}^{r}$, we obtain interval estimates of $t$ and $b$. We do not further detail this part since there is no difficulty.

\subsubsection{Second elements of $X^{++}$}

We decrease a little bit the values on criteria $j^{+}$and $j^{-}$, leading to $x_{j^{+}}^{\prime} \in X_{j^{+}}^{++}$ and $x_{j^{-}}^{\prime} \in X_{j^{-}}^{++}$such that $x_{j^{+}}^{\prime} \prec_{j^{+}} x_{j^{+}}$and $x_{j^{-}}^{\prime} \prec_{j^{-}} x_{j^{-}}$. Without loss of generality, we assume that $u_{j^{+}}^{\star}\left(x_{j^{+}}^{\prime}\right) \leq u_{j^{-}}^{\star}\left(x_{j^{-}}^{\prime}\right)$.

We run Alg1. From Lemma 3, Alg1 leads also to one transition, and it is located in the same part as for Section 4.2.1. Alg1 gives thus one single transition with a value in the estimated interval $\left(\underline{x_{i}}{ }^{\prime},{\overline{x_{i}}}^{\prime}\right)$.

If $\varepsilon$ is small enough, one shall have only two cases :

- If $x_{i}^{\prime} \prec_{i} \underline{x}_{i}$ and $\overline{x_{i}^{\prime}} \prec_{i} \overline{x_{i}}$, then the value of the transition decreases when $x_{j^{+}}, x_{j^{-}}$decrease. Hence by Lemma 3 , we conclude that the transition is the right one. Therefore we have by (18)

$$
\left\{\begin{array}{l}
u_{j^{+}}^{\star}\left(x_{j^{+}}\right)-t \in\left(\frac{1-t}{1-b}\left(u_{i}^{\star}\left(\underline{x_{i}}\right)-b\right), \frac{1-t}{1-b}\left(u_{i}^{\star}\left(\overline{x_{i}}\right)-b\right)\right) \\
\left.u_{j^{+}}^{\star}\left(x_{j^{+}}^{\prime}\right)-t \in\left(\frac{1-t}{1-b}\left(u_{i}^{\star} \underline{\left(x_{i}^{\prime}\right.}\right)-b\right), \frac{1-t}{1-b}\left(u_{i}^{\star}\left(\overline{x_{i}^{\prime}}\right)-b\right)\right)
\end{array}\right.
$$

Consider once more the case where there is no uncertainty. For attractive value of $z_{j^{+}} \in X_{j^{+}}$, let us denote by $\chi_{i}\left(z_{j^{+}}\right) \in X_{i}$ the right 
transition. Hence

$$
u_{j^{+}}^{\star}\left(z_{j^{+}}\right)=\frac{1-t}{1-b}\left(u_{i}^{\star}\left(\chi_{i}\left(z_{j^{+}}\right)\right)-b\right)+t=\frac{1-t}{1-b} u_{i}^{\star}\left(\chi_{i}\left(z_{j^{+}}\right)\right)+\frac{t-b}{1-b}
$$

From the results of $\operatorname{Alg} 1\left(x_{-i}\right)$ and $\operatorname{Alg} 1\left(x_{-i}^{\prime}\right)$, we obtain two values $\chi_{i}\left(z_{j^{+}}\right)$and $\chi_{i}\left(z_{j^{+}}^{\prime}\right)$. We deduce $\alpha_{i}$ and $\beta_{i}$ such that for $z_{j^{+}} \in\left\{x_{j^{+}}, x_{j^{+}}^{\prime}\right\}$

$$
u_{j^{+}}^{\star}\left(z_{j^{+}}\right)=\alpha_{i} u_{i}^{\star}\left(\chi_{i}\left(z_{j^{+}}\right)\right)+\beta_{i} .
$$

Hence, this gives $\alpha_{i}=\frac{1-t}{1-b}$ and $\beta_{i}=\frac{t-b}{1-b}$. Hence $\beta_{i}=1-\alpha_{i}$ and

$$
1-t=\alpha_{i} \times(1-b) \text {. }
$$

- If $x_{i}^{\prime} \succ_{i} \underline{x_{i}}$ and $\overline{x_{i}^{\prime}} \succ_{i} \overline{x_{i}}$, then the value of the transition increases when $x_{j^{+}}, x_{j^{-}}$decrease. Hence by Lemma 3 , we conclude that the transition is the left one. Therefore we have by (17)

$$
\left\{\begin{array}{l}
t-u_{j^{+}}^{\star}\left(x_{j^{+}}\right) \in\left(\frac{t}{b}\left(u_{i}^{\star}\left(\underline{x_{i}}\right)-b\right), \frac{t}{b}\left(u_{i}^{\star}\left(\overline{x_{i}}\right)-b\right)\right) \\
t-u_{j^{+}}^{\star}\left(x_{j^{+}}^{\prime}\right) \in\left(\frac{t}{b}\left(u_{i}^{\star}\left(\underline{x_{i}^{\prime}}\right)-b\right), \frac{t}{b}\left(u_{i}^{\star}\left(\overline{x_{i}^{\prime}}\right)-b\right)\right)
\end{array}\right.
$$

Consider once more the case where there is no uncertainty. Hence

$$
u_{j^{+}}^{\star}\left(z_{j^{+}}\right)=t-\frac{t}{b}\left(u_{i}^{\star}\left(\chi_{i}\left(z_{j^{+}}\right)\right)-b\right)=-\frac{t}{b} u_{i}^{\star}\left(\chi_{i}\left(z_{j^{+}}\right)\right)+2 t
$$

From the two answers obtained in Sections 4.2.1 and 4.2.2, we deduce $\alpha_{i}$ and $\beta_{i}$ such that

$$
u_{j^{+}}^{\star}\left(z_{j^{+}}\right)=-\alpha_{i} u_{i}^{\star}\left(\chi_{i}\left(z_{j^{+}}\right)\right)+\beta_{i}
$$

This gives $\alpha_{i}=\frac{t}{b}$ and $\beta_{i}=2 t$. We obtain

$$
t=\frac{\beta_{i}}{2} \quad, \quad b=\frac{\beta_{i}}{2 \alpha_{i}} .
$$

\subsubsection{First elements of $X^{--}$}

We continue the questionnaire with $y_{j^{+}} \in X_{j^{+}}^{++}$and $y_{j^{-}} \in X_{j^{-}}^{++}$. Without loss of generality, we assume that $u_{j^{+}}^{\star}\left(y_{j^{+}}\right) \leq u_{j^{-}}^{\star}\left(y_{j^{-}}\right)$. Alg1 is applied on these values. One obtains either one single transition with a value in the estimated interval $\left(\underline{y_{i}}, \overline{y_{i}}\right)$, or two transitions with values in the estimated intervals $\left(\underline{y_{i}^{l}}, \overline{y_{i}^{l}}\right)$ and $\left(\underline{y_{i}^{r}}, \overline{y_{i}^{r}}\right)$ for the left and right transitions respectively. 


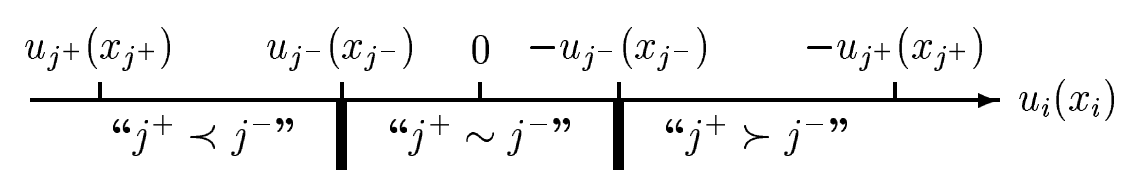

Figure 5: The two transitions.

If there is only one transition, we go to Section 4.2.4. We consider thus here the case of a double transition.

The exact left transition $y_{i}^{l}$ satisfies $u_{i}\left(y_{i}^{l}\right) \in\left(u_{i}\left(\underline{y_{i}^{l}}\right), u_{i}\left(\overline{y_{i}^{l}}\right)\right)$. From the third case of Lemma $3, u_{i}\left(y_{i}^{l}\right)=u_{j^{-}}\left(y_{j^{-}}\right)$. Hence $u_{j^{-}}\left(y_{j^{-}}\right) \in\left(u_{i}\left(\underline{y_{i}^{l}}\right), u_{i}\left(\overline{y_{i}^{l}}\right)\right)$. Since $\underline{y_{i}^{l}}$ and $\overline{y_{i}^{l}}$ are repulsive, one has $u_{i}\left(\underline{y_{i}^{l}}\right)=\frac{t}{b}\left(u_{i}^{\star}\left(\underline{y_{i}^{l}}\right)-b\right)$ and $u_{i}\left(\overline{y_{i}^{l}}\right)=$ $\frac{t}{b}\left(u_{i}^{\star}\left(\overline{y_{i}^{l}}\right)-b\right)$. Thus

$$
u_{j^{-}}^{\star}\left(y_{j^{-}}\right)-t \in\left(\frac{t}{b}\left(u_{i}^{\star}\left(\underline{y_{i}^{l}}\right)-b\right), \frac{t}{b}\left(u_{i}^{\star}\left(\overline{y_{i}^{l}}\right)-b\right)\right)
$$

The exact right transition $y_{i}^{r}$ satisfies $u_{i}\left(y_{i}^{r}\right) \in\left(u_{i}\left(\underline{y_{i}^{r}}\right), u_{i}\left(\overline{y_{i}^{r}}\right)\right)$. From the third case of Lemma $3, u_{i}\left(y_{i}^{r}\right)=-u_{j^{-}}\left(y_{j^{-}}\right)$. Hence $-u_{j^{-}}\left(y_{j^{-}}\right) \in\left(u_{i}\left(\underline{y_{i}^{r}}\right), u_{i}\left(\overline{y_{i}^{r}}\right)\right)$. Since $y_{i}^{r}$ and $\overline{y_{i}^{r}}$ are attractive, one obtains

$$
t-u_{j^{-}}^{\star}\left(y_{j^{-}}\right) \in\left(\frac{1-t}{1-b}\left(u_{i}^{\star}\left(\underline{y_{i}^{r}}\right)-b\right), \frac{1-t}{1-b}\left(u_{i}^{\star}\left(\overline{y_{i}^{r}}\right)-b\right)\right)
$$

Consider first the case when there is no uncertainty

$$
\left\{\begin{array}{l}
\frac{t}{b}\left(u_{i}^{\star}\left(y_{i}^{l}\right)-b\right)=u_{j^{-}}^{\star}\left(y_{j^{-}}\right)-t \\
\frac{1-t}{1-b}\left(u_{i}^{\star}\left(y_{i}^{r}\right)-b\right)=t-u_{j^{-}}^{\star}\left(y_{j^{-}}\right)
\end{array}\right.
$$

which gives

$$
\left\{\begin{array}{l}
t=\frac{u_{j^{-}}^{\star}\left(y_{j^{-}}\right) \times b}{u_{i}^{\star}\left(y_{i}^{l}\right)}=: f_{3}(b) \\
t=\frac{-\left(1+u_{j^{-}}^{\star}\left(y_{j-}\right)\right) \times b+u_{j-}^{\star}\left(y_{j-}\right)+u_{i}^{\star}\left(y_{i}^{r}\right)}{1+u_{i}^{\star}\left(y_{i}^{r}\right)-2 b}=: f_{4}(b)
\end{array}\right.
$$

Function $f_{3}$ is affine and increasing. In addition, $f_{3}(0)=0$. One has

$$
f_{4}^{\prime}(b)=-\frac{\left(1-u_{j^{-}}^{\star}\left(y_{j^{-}}\right)\right)\left(1-u_{i}^{\star}\left(y_{i}^{l}\right)\right)}{\left(1+u_{i}^{\star}\left(y_{i}^{r}\right)-2 b\right)^{2}}<0 .
$$

$f_{4}$ is an hyperbolic curve with the $x$-asymptote at value $\frac{1+u_{i}^{\star}\left(y_{i}^{l}\right)}{2} \in(0,1)$ and the $y$-asymptote at value $\frac{1+u_{j^{-}}^{\star}\left(y_{j^{-}}\right)}{2} \in(0,1)$. Moreover, $f_{4}$ goes through point 


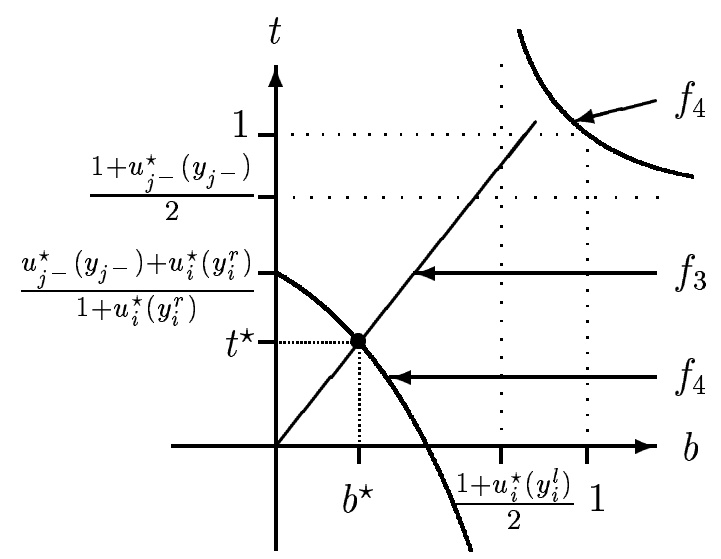

Figure 6: Functions $f_{3}$ and $f_{4}$.

$(b, t)=(1,1)$. Hence, the upper right part of $f_{4}$ does not intersect the open square $(0,1)^{2}$.

We conclude that $f_{3}$ and $f_{4}$ intersect exactly at one point $\left(b^{\star}, t^{\star}\right) \in(0,1)^{2}$ (see Fig. 6). Hence there is a single solution to our problem.

When we take into account the uncertainties in $y_{i}^{l}$ and $y_{i}^{r}$, we obtain interval estimates of $t$ and $b$. We do not further detail this part since there is no difficulty.

\subsubsection{Second elements of $X^{--}$}

We increase a little bit the values on criteria $j^{+}$and $j^{-}$, leading to $y_{j^{+}}^{\prime} \in X_{j^{+}}^{++}$ and $y_{j^{-}}^{\prime} \in X_{j^{-}}^{++}$such that $y_{j^{+}}^{\prime} \succ_{j^{+}} y_{j^{+}}$and $y_{j^{-}}^{\prime} \succ_{j^{-}} y_{j^{-}}$. Without loss of generality, we assume that $u_{j^{+}}^{\star}\left(y_{j^{+}}^{\prime}\right) \leq u_{j^{-}}^{\star}\left(y_{j^{-}}^{\prime}\right)$.

We run Alg1. From Lemma 3, Alg1 leads also to one transition, and it is located in the same part as for Section 4.2.3. Alg1 gives thus one single transition with a value in the estimated interval $\left(\underline{y_{i}},{\overline{y_{i}}}^{\prime}\right)$.

If $\varepsilon$ is small enough, one shall have only two cases :

- If $\underline{y_{i}^{\prime}} \prec_{i} \underline{y_{i}}$ and $\overline{y_{i}^{\prime}} \prec_{i} \overline{y_{i}}$, then the value of the transition decreases when $y_{j^{+}}, y_{j^{-}}$increase. Hence by Lemma 3, we conclude that the transition is the right one. Therefore we have by (22)

$$
\left\{\begin{array}{l}
t-u_{j^{-}}^{\star}\left(y_{j^{-}}\right) \in\left(\frac{1-t}{1-b}\left(u_{i}^{\star}\left(\underline{y_{i}}\right)-b\right), \frac{1-t}{1-b}\left(u_{i}^{\star}\left(\overline{y_{i}}\right)-b\right)\right) \\
t-u_{j^{-}}^{\star}\left(x_{j^{-}}^{\prime}\right) \in\left(\frac{1-t}{1-b}\left(u_{i}^{\star}\left(\underline{y_{i}^{\prime}}\right)-b\right), \frac{1-t}{1-b}\left(u_{i}^{\star}\left(\overline{y_{i}^{\prime}}\right)-b\right)\right)
\end{array}\right.
$$

Consider once more the case where there is no uncertainty. For repulsive value of $z_{j^{+}} \in X_{j^{+}}$, let us denote by $\chi_{i}\left(z_{j^{+}}\right) \in X_{i}$ the right 
transition. Hence

$$
u_{j^{-}}^{\star}\left(z_{j^{-}}\right)=t-\frac{1-t}{1-b}\left(u_{i}^{\star}\left(\chi_{i}\left(z_{j^{+}}\right)\right)-b\right)=-\frac{1-t}{1-b} u_{i}^{\star}\left(\chi_{i}\left(z_{j^{+}}\right)\right)+\frac{b(1-t)+t(1-b)}{1-b}
$$

From the two answers obtained in Sections 4.2.3 and 4.2.4, we deduce $\alpha_{i}^{\prime}$ and $\beta_{i}^{\prime}$ such that

$$
u_{j^{+}}^{\star}\left(z_{j^{+}}\right)=-\alpha_{i}^{\prime} u_{i}^{\star}\left(\chi_{i}\left(z_{j^{+}}\right)\right)+\beta_{i}^{\prime}
$$

This gives $\alpha_{i}^{\prime}=\frac{1-t}{1-b}$ and $\beta_{i}^{\prime}=\frac{b(1-t)+t(1-b)}{1-b}$. We obtain

$$
b=\frac{\alpha_{i}^{\prime}+\beta_{i}^{\prime}-1}{2 \alpha_{i}^{\prime}} \quad, \quad t=\frac{\beta_{i}^{\prime}-\alpha_{i}^{\prime}-1}{2} .
$$

- If $\underline{x_{i}^{\prime}} \succ_{i} \underline{x_{i}}$ and $\overline{x_{i}^{\prime}} \succ_{i} \overline{x_{i}}$, then the value of the transition increases when $x_{j^{+}}, x_{j^{-}}$increase. Hence by Lemma 3 , we conclude that the transition is the left one. Therefore we have by $(21)$

$$
\left\{\begin{array}{l}
u_{j^{-}}^{\star}\left(y_{j^{-}}\right)-t \in\left(\frac{t}{b}\left(u_{i}^{\star}\left(\underline{y_{i}}\right)-b\right), \frac{t}{b}\left(u_{i}^{\star}\left(\overline{y_{i}}\right)-b\right)\right) \\
u_{j^{+}}^{\star}\left(x_{j^{+}}^{\prime}\right)-t \in\left(\frac{t}{b}\left(u_{i}^{\star}\left(\underline{y_{i}^{\prime}}\right)-b\right), \frac{t}{b}\left(u_{i}^{\star}\left(\overline{y_{i}^{\prime}}\right)-b\right)\right)
\end{array}\right.
$$

Consider once more the case where there is no uncertainty. Hence

$$
u_{j^{+}}^{\star}\left(z_{j^{+}}\right)=\frac{t}{b}\left(u_{i}^{\star}\left(\chi_{i}\left(z_{j^{+}}\right)\right)-b\right)+t=\frac{t}{b} u_{i}^{\star}\left(\chi_{i}\left(z_{j^{+}}\right)\right)
$$

From the two answers obtained in Sections 4.2.3 and 4.2.4, we deduce $\alpha_{i}^{\prime}$ such that

$$
u_{j^{+}}^{\star}\left(z_{j^{+}}\right)=\alpha_{i}^{\prime} u_{i}^{\star}\left(\chi_{i}\left(z_{j^{+}}\right)\right)
$$

This gives $\alpha_{i}^{\prime}=\frac{t}{b}$. We obtain

$$
t=\alpha_{i}^{\prime} b
$$

\subsubsection{Summary}

In all cases, except for the first case of Section 4.2.2 and the second case of Section 4.2.4, we obtain a unique solution. In this special case, we have both (19) and (24). This gives if $\alpha_{i}^{\prime} \neq \alpha_{i}$

$$
b=\frac{1-\alpha_{i}}{\alpha_{i}^{\prime}-\alpha_{i}} \quad, \quad b=\alpha_{i}^{\prime} \times b .
$$

If $\alpha_{i}^{\prime}=\alpha_{i}$, then necessarily one has $\alpha_{i}^{\prime}=\alpha_{i}=1$ so that $t=b$. This case corresponds to the case where there is no special perception on attribute $X_{i}$. We deal this case in the following section. 


\subsection{Determination of the neutral element}

We assume that (15) holds for all $k \in N$. This implies that $b=t$. We propose a technique for determining $t$ under the assumption that all attributes $X_{k}$ are cardinal and correspond to an interval.

Let $\epsilon>0$. Let $x \in X$ such that

$$
\forall l \neq m \quad\left|u_{l}^{\star}\left(x_{l}\right)-u_{m}^{\star}\left(x_{m}\right)\right| \geq \epsilon \text { and } \forall l\left|u_{l}^{\star}\left(x_{l}\right)-t\right| \geq \epsilon
$$

We assume that

$$
u_{j^{+}}^{\star}\left(x_{j^{+}}\right)<u_{i}^{\star}\left(x_{i}\right)<u_{j^{-}}^{\star}\left(x_{j^{-}}\right) .
$$

Define $x^{\epsilon}$ by

$$
\left(x^{\epsilon}\right)_{m}= \begin{cases}u_{j^{+}}^{\star-1}\left(u_{j^{+}}^{\star}\left(x_{j^{+}}\right)+\frac{\epsilon}{2}\right) & \text { if } m=j^{+} \\ u_{j^{-}}^{\star}\left(u_{j^{-}}^{\star}\left(x_{j^{-}}\right)-\frac{\epsilon}{2}\right) & \text { if } m=j^{-} \\ x_{m} \text { otherwise } & \end{cases}
$$

We need the following simple result.

Lemma 4 If $\left|u_{m}^{\star}\left(x_{m}\right)-t\right| \leq\left|u_{l}^{\star}\left(x_{l}\right)-t\right|$ and $u_{m}^{\star}\left(x_{m}\right)>u_{l}^{\star}\left(x_{l}\right)$ then $t \geq$ $\frac{u_{m}^{\star}\left(x_{m}\right)+u_{l}^{\star}\left(x_{l}\right)}{2}$.

If $\left|u_{m}^{\star}\left(x_{m}\right)-t\right| \leq\left|u_{l}^{\star}\left(x_{l}\right)-t\right|$ and $u_{m}^{\star}\left(x_{m}\right)<u_{l}^{\star}\left(x_{l}\right)$ then $t \leq \frac{u_{m}^{\star}\left(x_{m}\right)+u_{l}^{\star}\left(x_{l}\right)}{2}$.

Proof : The proof of this result is obvious and left to the reader.

If $u_{i}^{\star}\left(x_{i}\right)>t$ and $\left|u_{i}^{\star}\left(x_{i}\right)-t\right| \geq\left|u_{j^{+}}^{\star}\left(x_{j^{+}}\right)-t\right|$, then by Lemma 4

$$
t \leq \frac{u_{j^{+}}^{\star}\left(x_{j^{+}}\right)+u_{i}^{\star}\left(x_{i}\right)}{2} \leq \frac{u_{j^{+}}^{\star}\left(x_{j^{+}}^{\epsilon}\right)+u_{i}^{\star}\left(x_{i}^{\epsilon}\right)}{2}
$$

Hence $\left|u_{i}^{\star}\left(x_{i}^{\epsilon}\right)-t\right| \geq\left|u_{j^{+}}^{\star}\left(x_{j^{+}}^{\epsilon}\right)-t\right|$. We conclude by (25) that the conditions of the first part of (S3) are fulfilled for both $x$ and $x^{\epsilon}$. Therefore

$$
x^{\epsilon} \succ x
$$

If $u_{i}^{\star}\left(x_{i}\right)<t$ and $\left|u_{i}^{\star}\left(x_{i}\right)-t\right| \leq\left|u_{j^{-}}^{\star}\left(x_{j^{-}}\right)-t\right|$, then by Lemma 4

$$
t \geq \frac{u_{j^{-}}^{\star}\left(x_{j^{-}}\right)+u_{i}^{\star}\left(x_{i}\right)}{2} \leq \frac{u_{j^{-}}^{\star}\left(x_{j^{-}}^{\epsilon}\right)+u_{i}^{\star}\left(x_{i}^{\epsilon}\right)}{2} .
$$


Hence $\left|u_{i}^{\star}\left(x_{i}^{\epsilon}\right)-t\right| \geq\left|u_{j^{-}}^{\star}\left(x_{j^{-}}^{\epsilon}\right)-t\right|$. By (25), the conditions of the second part of (S3) are fulfilled for both $x$ and $x^{\epsilon}$. Therefore

$$
x^{\epsilon} \prec x
$$

If criterion $i$ is the closest to the neutral level among criteria $i, j^{+}, j^{-}$, then the three cases $x^{\epsilon} \succ x, x^{\epsilon} \prec x$ and $x^{\epsilon} \sim x$ are possible. The case of indifference between $x$ and $x^{\epsilon}$ necessarily happens when statement (S3) cannot be applied. This implies that we have $\left|u_{i}^{\star}\left(x_{i}\right)-t\right| \leq \min \left(\left|u_{j^{+}}^{\star}\left(x_{j^{+}}\right)-t\right|,\left|u_{j^{-}}^{\star}\left(x_{j^{-}}\right)-t\right|\right)$. Hence

$$
\text { If } x^{\epsilon} \sim x \text { then }\left|u_{i}^{\star}\left(x_{i}\right)-t\right| \leq \min \left(\left|u_{j^{+}}^{\star}\left(x_{j^{+}}\right)-t\right|,\left|u_{j^{-}}^{\star}\left(x_{j^{-}}\right)-t\right|\right)
$$

We propose Algorithm 2 for the assessment of neutral level $t$. It is based on the comparison that the actor makes between successive pairs of acts $x$, $x^{\epsilon}$ for which the coordinates w.r.t. criteria $i, j^{+}$and $j^{-}$tend to the neutral value. The value of these coordinates are determined in the algorithm. This is a kind of dichotomy method, except that the interval is split into three subintervals instead of two as in the original dichotomy method. Let $x_{-i, j^{+}, j^{-}}$ fixed.

Algorithm 2. Determination of $t$.

Step 1. We start with two a-priori bounds $\underline{t}_{0}$ and $\bar{t}_{0}$ of $t$ such that

$$
t \in\left[\underline{t}_{0}, \bar{t}_{0}\right] .
$$

We set $\underline{t}=\underline{t}_{0}$ and $\bar{t}=\bar{t}_{0}$. Set $p=0$.

Step 2. Consider $x_{i}, x_{j^{+}}, x_{j^{-}}$such that $u_{j^{+}}^{\star}\left(x_{j^{+}}\right)=\frac{5}{6} \underline{t}_{p}+\frac{1}{6} \bar{t}_{p}, \quad u_{i}^{\star}\left(x_{i}\right)=\frac{1}{2} \underline{t}_{p}+\frac{1}{2} \bar{t}_{p} \quad$ and $\quad u_{j^{-}}^{\star}\left(x_{j^{-}}\right)=\frac{1}{6} \underline{t}_{p}+\frac{5}{6} \bar{t}_{p}$.

We obtain in this case $\epsilon_{p}=\frac{\bar{t}_{p}-\underline{\underline{t}}}{3}$.

Step 3. The actor is asked to compare $x$ with $x^{\epsilon}$. We have three cases

- If $x^{\epsilon} \succ x$ then we set

$$
\bar{t}_{p+1}=\frac{u_{i}^{\star}\left(x_{i}\right)+u_{j^{-}}^{\star}\left(x_{j^{-}}\right)}{2} .
$$


- If $x^{\epsilon} \prec x$ then we set

$$
\underline{t}_{p+1}=\frac{u_{i}^{\star}\left(x_{i}\right)+u_{j^{+}}^{\star}\left(x_{j^{+}}\right)}{2} .
$$

- If $x^{\epsilon} \sim x$ then we set

$$
\underline{t}_{p+1}=\frac{u_{i}^{\star}\left(x_{i}\right)+u_{j^{+}}^{\star}\left(x_{j^{+}}\right)}{2}, \bar{t}_{p+1}=\frac{u_{i}^{\star}\left(x_{i}\right)+u_{j^{-}}^{\star}\left(x_{j^{-}}\right)}{2} .
$$

Step 4. If $\bar{t}_{p+1}-\underline{t}_{p+1}$ is greater than a given threshold $\eta$, then set $p \leftarrow p+1$ and goto Step 2 .

Lemma 5 The exact value $t$ of $u_{i}^{\star}\left(\mathbf{0}_{i}\right)$ always belongs to the interval $\left[\underline{t}_{p}, \bar{t}_{p}\right]$ at each iteration $p$ of Algorithm 2. In addition, the size of the interval $\left[\underline{t}_{p}, \bar{t}_{p}\right]$ is reduced at least by a factor $\frac{2}{3}$ at each iteration of the algorithm.

Proof : First, from the choice of $x_{i}, x_{j^{+}}, x_{j^{-}}$in Step 2 of Algorithm 2, we have that

$$
u_{i}^{\star}\left(x_{i}\right), u_{j^{+}}^{\star}\left(x_{j^{+}}\right), u_{j^{-}}^{\star}\left(x_{j^{-}}\right) \in\left[\underline{t}_{p}, \bar{t}_{p}\right]
$$

and

$$
u_{j^{+}}^{\star}\left(x_{j^{+}}\right)<u_{i}^{\star}\left(x_{i}\right)<u_{j^{-}}^{\star}\left(x_{j^{-}}\right)
$$

at all iterations of Algorithm 2.

We consider now Step 3. We have three cases.

- Consider first the case when $x^{\epsilon} \succ x . x^{\epsilon} \succ x$ occurs either when the conditions of the first part of (S3) are fulfilled, or when criterion $i$ is the closest to the neutral level among criteria $i, j^{+}, j^{-}$. Hence, we have either

$u_{i}^{\star}\left(x_{i}\right)>t$ and $\left(\left|u_{i}^{\star}\left(x_{i}\right)-t\right| \geq\left|u_{j^{+}}^{\star}\left(x_{j^{+}}\right)-t\right|\right.$ or $\left.\left|u_{i}^{\star}\left(x_{i}\right)-t\right| \geq\left|u_{j^{-}}^{\star}\left(x_{j^{-}}\right)-t\right|\right)$

or

$$
\left|u_{i}^{\star}\left(x_{i}\right)-t\right| \leq \min \left(\left|u_{j^{+}}^{\star}\left(x_{j^{+}}\right)-t\right|,\left|u_{j^{-}}^{\star}\left(x_{j^{-}}\right)-t\right|\right)
$$

Consider first the case when (29) holds. By Lemma 4 and (25), we get

$$
\frac{u_{i}^{\star}\left(x_{i}\right)+u_{j^{+}}^{\star}\left(x_{j^{+}}\right)}{2} \leq t \leq \frac{u_{i}^{\star}\left(x_{i}\right)+u_{j^{-}}^{\star}\left(x_{j^{-}}\right)}{2}
$$

Consider now the case when (28) holds. By Lemma 4, we have either $t \geq \frac{u_{i}^{\star}\left(x_{i}\right)+u_{j^{-}}^{\star}\left(x_{j^{-}}\right)}{2}$ or $t \leq \frac{u_{i}^{\star}\left(x_{i}\right)+u_{j^{+}}^{\star}\left(x_{j+}\right)}{2}$. Since $t<u_{i}^{\star}\left(x_{i}\right)$, we obtain

$$
t \leq \frac{u_{i}^{\star}\left(x_{i}\right)+u_{j^{+}}^{\star}\left(x_{j^{+}}\right)}{2} .
$$


Combining previous two relations, we get

$$
t \leq \frac{u_{i}^{\star}\left(x_{i}\right)+u_{j^{-}}^{\star}\left(x_{j^{-}}\right)}{2} .
$$

Hence $t \leq \bar{t}_{p+1}$. From (27), $\bar{t}_{p+1}$ is strictly lower than $\bar{t}_{p}$.

- Consider now the case when $x^{\epsilon} \prec x$. We have either

$$
u_{i}^{\star}\left(x_{i}\right)<t \text { and }\left(\left|u_{i}^{\star}\left(x_{i}\right)-t\right| \geq\left|u_{j^{+}}^{\star}\left(x_{j^{+}}\right)-t\right| \text { or }\left|u_{i}^{\star}\left(x_{i}\right)-t\right| \geq\left|u_{j^{-}}^{\star}\left(x_{j^{-}}\right)-t\right|\right)
$$

or

$$
\left|u_{i}^{\star}\left(x_{i}\right)-t\right| \leq \min \left(\left|u_{j^{+}}^{\star}\left(x_{j^{+}}\right)-t\right|,\left|u_{j^{-}}^{\star}\left(x_{j^{-}}\right)-t\right|\right)
$$

We proceed as previously. Consider first the case when (31) holds. We get by Lemma 4

$$
\frac{u_{i}^{\star}\left(x_{i}\right)+u_{j^{+}}^{\star}\left(x_{j^{+}}\right)}{2} \leq t \leq \frac{u_{i}^{\star}\left(x_{i}\right)+u_{j^{-}}^{\star}\left(x_{j^{-}}\right)}{2} .
$$

Consider now the case when (30) holds. By Lemma 4, we have either $t \geq \frac{u_{i}^{\star}\left(x_{i}\right)+u_{j-}^{\star}\left(x_{j-}\right)}{2}$ or $t \leq \frac{u_{i}^{\star}\left(x_{i}\right)+u_{j+}^{\star}\left(x_{j}+\right)}{2}$. Since $t>u_{i}^{\star}\left(x_{i}\right)$, we obtain

$$
t \geq \frac{u_{i}^{\star}\left(x_{i}\right)+u_{j^{-}}^{\star}\left(x_{j^{-}}\right)}{2}
$$

Combining previous two relations, we get

$$
t \geq \frac{u_{i}^{\star}\left(x_{i}\right)+u_{j^{+}}^{\star}\left(x_{j^{+}}\right)}{2} .
$$

Hence $t \geq \underline{t}_{p+1}$. From (27), $\underline{t}_{p+1}$ is strictly greater than $\underline{t}_{p}$.

- The last case is $x^{\epsilon} \sim x$. By (26) and Lemma 4, we get

$$
\frac{u_{i}^{\star}\left(x_{i}\right)+u_{j^{+}}^{\star}\left(x_{j^{+}}\right)}{2} \leq t \leq \frac{u_{i}^{\star}\left(x_{i}\right)+u_{j^{-}}^{\star}\left(x_{j^{-}}\right)}{2} .
$$

Hence $\underline{t}_{p+1} \leq t \leq \bar{t}_{p+1}$. From (27), $\bar{t}_{p+1}$ is strictly lower than $\bar{t}_{p}$, and $\underline{t}_{p+1}$ is strictly greater than $\underline{t}_{p}$.

Since $\frac{u_{i}^{\star}\left(x_{i}\right)+u_{j+}^{\star}\left(x_{j}+\right)}{2}=\frac{2}{3} \underline{t}_{p}+\frac{1}{3} \bar{t}_{p}$, and $\frac{u_{i}^{\star}\left(x_{i}\right)+u_{j-}^{\star}\left(x_{j-}\right)}{2}=\frac{1}{3} \underline{t}_{p}+\frac{2}{3} \bar{t}_{p}$, we have

$$
\bar{t}_{p+1}-\underline{t}_{p+1} \leq \frac{2}{3}\left(\bar{t}_{p}-\underline{t}_{p}\right)
$$


From Lemma 5 , we conclude that interval $[\underline{t}, \bar{t}]$ converges to value $t$. More precisely, precision $\eta$ is reached in, at most, $\frac{\log \left(\eta /\left(\bar{t}_{0}-\underline{t}_{0}\right)\right)}{\log 2 / 3}$ iterations.

Previous method can be carried over to the case of qualitative attributes. In this case, we replace the comparison of $x^{\epsilon}$ with $x$, by question Q1.

\section{References}

[1] C.A. Bana e Costa, and J.C. Vansnick. A theoretical framework for Measuring Attractiveness by a Categorical Based Evaluation TecHnique (Macbeth). In Proc. XIth It. Cone. on Multi Criteria Decision Making, Portugal, 1994, pip.15-24.

[2] C.A. Bana e Costa, and J.C. Vans-nick. Preference relations and MCDM. In: Multi Criteria Decision Making: advances in MCDM models, algorithms, theory and applications. T. Gal, T. Steward, T. Hanna Feds. Kluwer, 1999.

[3] C.A. Bana e Costa, and J.C. Vansnick. Applications of the MACBETH approach in the framework of an additive aggregation model. J. of MultiCriteria Decision Analysis 6 (1997) 107-114.

[4] C.A. Bana e Costa, F. Nunes da Silva, and J.C. Vansnick. Conflict dissolution in the public sector: A case-study. European Journal of Operational Research 130 (2001) 388-401.

[5] C. Boutilier, R.I. Brafman, C. Domshlak, H.H. Hoos and D. Poole. CPnets: a tool for representing and reasoning with conditional Ceteris Paribus preference statements. J. of Artificial Intelligence Research 21 (2004) 135-191.

[6] D. Bouyssou, M. Pirlot. Preferences for multi-attributed alternatives: traces, dominance, and numerical representations. J. of Mathematical Psychology 48 (2004) 167-185.

[7] R. Brafman, C. Domshlak. Introducing variable importance tradeoffs into CP-nets. AIPS'02 Workshop on Planning and Scheduling with Multiple Criteria.

[8] G. Choquet, Theory of capacities, Annales de l'Institut Fourier 5 (1953) 131-295. 
[9] F. De Finetti. Theory of Probability. Wiley, London, 1974.

[10] J.C. Fodor and M. Roubens. Fuzzy preferences modeling and multicriteria decision aid. Kluwer Academic Publisher, 1994.

[11] M. Grabisch, Ch. Labreuche \& J.C. Vansnick. On the Extension of Pseudo-Boolean Functions for the Aggregation of Interacting Criteria, European Journal of Operational Research 148 (2003) 28-47.

[12] M. Grabisch, T. Murofushi and M. Sugeno, Fuzzy measures and integrals, Physica-Verlag, Heidelberg, New York, 2000.

[13] M. Grabisch, Ch. Labreuche. Bi-Capacities for decision making on bipolar scales. In: EUROFUSE Workshop on Information Systems, Varenna, Italy, September 2002.

[14] D. Kahneman, D. Tversky. Propect theory: an analysis of decision under risk. Econometrica 47 (1979) 263-291.

[15] R.L. Keeney, H. Raiffa, Decision with Multiple Objectives, Wiley, New York, 1976.

[16] D.H. Krantz, R.D. Luce, P. Suppes, A. Tversky, Foundations of measurement, vol 1: Additive and Polynomial Representations, Academic Press, San Diego, 1971.

[17] Ch. Labreuche, M. Grabisch. The Choquet integral for the aggregation of interval scales in multicriteria decision making, Fuzzy Sets and Systems 137 (2003) 11-26.

[18] C. Labreuche, M. Grabisch. Generalized Choquet-like aggregation functions for handling bipolar scales. European J. of Operational Research, to appear.

[19] J. Pennings, A. Smidts. Assessing the construct validity of risk attitude. Management Science 46 (2000) 1337-1348 .

[20] Nicolas Rescher, An introduction to value theory.

[21] H. Simon. Rational choice and the structure of the environment. Psychological Review 63(2) (1956) 129-138.

[22] P. Slovic, M. Finucane, E. Peters, D.G. MacGregor, The affect heuristic. in: T. Gilovitch, D. Griffin, D. Kahneman (Eds.), Heuristics and biases: the psychology of intuitive judgment, Cambridge University Press, 2002, pp. $397-420$. 